\title{
Planetary nebulae in M 32 and the bulge of M 31: Line intensities and oxygen abundances
}

\author{
M.G. Richer ${ }^{1, \star, \star \star}$, G. Stasińska ${ }^{2, \star \star}$, and M.L. McCall ${ }^{3}$ \\ 1 Instituto de Astronomía, UNAM, Apartado Postal 70-264, 04510 Mexico D. F., Mexico \\ e-mail: richer@astroscu.unam.mx \\ 2 DAEC, Observatoire de Meudon, 5 place Jules Janssen, F-92195 Meudon Cedex, France \\ e-mail: grazyna@obspm.fr \\ 3 Dept. of Physics and Astronomy, York University, 4700 Keele Street, Toronto, Ontario, Canada M3J 1P3 \\ e-mail: mccall@aries.phys.yorku.ca
}

Received June 8; accepted September 22, 1998

\begin{abstract}
We present spectroscopy of planetary nebulae in M 32 and in the bulge of M 31 that we obtained with the MOS spectrograph at the Canada-France-Hawaii Telescope. Our sample includes 30 planetary nebulae in M 31 and 9 planetary nebulae in M 32. We also observed one $\mathrm{H}$ II region in the disk of $\mathrm{M} 31$. We detected [O III] $\lambda 4363$ in 18 of the planetary nebulae, 4 in M 32 and 14 in the bulge of M 31. We use our line intensities to derive electron temperatures and oxygen abundances for the planetary nebulae.
\end{abstract}

Key words: galaxies: individual: M 31, M 32 galaxies abundances - galaxies: elliptical and lenticular galaxies: evolution - galaxies: ISM

\section{Introduction}

One of the most important clues concerning the early evolution of dynamically hot galaxies (DHGs: ellipticals, dwarf spheroidals, and bulges of spirals) in the fundamental plane of galaxies is the existence of a well-defined relationship between metallicity and mass (e.g., Bender et al. 1993). The fundamental lesson taught by this relation is that star formation in DHGs stopped because of gas loss, with less massive systems losing greater fractions of their

Send offprint requests to: M.G. Richer

* The overwhelming majority of my work on this project was done while I was at the Observatoire de Meudon as a member of the DAEC.

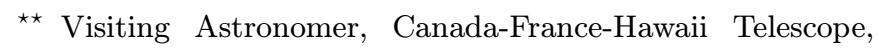
operated by the National Research Council of Canada, the Centre National de la Recherche Scientifique de France, and the University of Hawaii. gas. Outflow probably begins when supernovae have raised the internal energy of the gas enough to allow it to escape the potential well (e.g., Brocato et al. 1990).

Most commonly, the metallicity in DHGs is measured via the $\mathrm{Mg}_{2}$ index. While the $\mathrm{Mg}_{2}$ index is an excellent means of ranking galaxy metallicities, it does not yield an abundance directly, i.e., the number density of a particular element relative to hydrogen, and calibrations of the $\mathrm{Mg}_{2}$ index (model-dependent) are usually in terms of the iron abundance, an element whose production is notoriously difficult to model. Though this may be best for some purposes, e.g., studies of stellar populations, it is not sufficient for all purposes. To study the chemical evolution of DHGs requires the abundance of an element whose production is well understood. Were such abundances available, there would be some hope of quantifying the gas fraction at which DHGs of different masses begin to lose mass. Knowledge of the abundances would admit studying the yield of heavy elements, and hence the slope of the stellar initial mass function during the star formation epoch. Given the known photometric and dynamical properties of DHGs today, abundances would also allow us to study the global energetics involved during their star formation phase.

This paper is one of a sequence investigating the oxygen abundances of DHGs. Here, we present oxygen abundances for samples of planetary nebulae in M 32 and in the bulge of M 31. These two nearby systems are good representatives of typical DHGs. Though M 32's light profile may be truncated compared to isolated ellipticals, its structural, dynamical, and spectral properties are perfectly typical for an elliptical of its luminosity (Kormendy 1985; Bender et al. 1993). Similarly, recent work on the DHG fundamental plane has shown that the photometric, dynamical, and stellar population properties 
Table 1. Observing log

\begin{tabular}{|c|c|c|c|c|c|c|}
\hline Date & Object & Grism & Dispersion & Spectral Range $^{a}$ & Exposures & Standards \\
\hline 1994 Aug. 1/2 & M 32 & U900 & $0.9 \AA /$ pixel & $3727 \AA-\mathrm{H} \gamma$ & $1 \times 2700 \mathrm{~s}$ & Feige 15 \\
\hline 1994 Aug. 2/3 & NGC 6720 & U900 & $0.9 \AA /$ pixel & $3600 \AA-5400 \AA$ & $\begin{array}{l}3 \times 1500 \mathrm{~s} \\
1 \times 1200 \mathrm{~s}\end{array}$ & Feige 15 \\
\hline \multirow{4}{*}{1994 Aug. 3/4 } & M 32 & U900 & $0.9 \stackrel{\AA}{\AA} /$ pixel & $3727 \AA$ & $3 \times 2700 \mathrm{~s}$ & Feige 15 \\
\hline & M 32 & O300 & $3.5 \AA /$ pixel & $4686 \AA-1 \mu \mathrm{m}$ & $\begin{array}{l}1 \times 900 \mathrm{~s} \\
1 \times 1800 \mathrm{~s}\end{array}$ & Wolf 1346 \\
\hline & M 32 & $\mathrm{~B} 600$ & $1.5 \AA /$ pixel & $3727 \AA-5876 \AA$ & $1 \times 1800 \mathrm{~s}$ & $\begin{array}{l}\text { Wolf } 1346 \\
\text { Feige } 15\end{array}$ \\
\hline & M 31 & $\mathrm{~B} 600$ & $1.5 \AA /$ pixel & $3727 \AA-5876 \AA$ & $\begin{array}{l}3 \times 2700 \mathrm{~s} \\
1 \times 1200 \mathrm{~s}\end{array}$ & $\begin{array}{l}\text { Wolf } 1346 \\
\text { Feige } 15\end{array}$ \\
\hline
\end{tabular}

a These are minimum spectral ranges. The actual spectral range will depend upon the object's position within the spectrograph's field of view.

of bulges follow those of pure ellipticals (Bender et al. 1992, 1993).

Oxygen is an excellent element with which to study the evolution of galaxies. Oxygen is a primary element whose sole significant production site is type II supernovae (Wheeler et al. 1989), so its abundance is tied directly to the history of massive star formation, and the enrichment time scale is short compared to the gas consumption time scale. Oxygen abundances are also easily observable in planetary nebulae. Planetary nebulae have high electron temperatures, so the temperature-sensitive [O III $] \lambda 4363$ line is observable, making it possible to determine accurate electron temperatures in high metallicity environments. Further, the dominant ionization stages of oxygen, $\mathrm{O}^{+}$and $\mathrm{O}^{++}$, have observable lines, while other ionization stages are easily accounted for using ratios of readily detectable helium lines (e.g., Kingsburgh \& Barlow 1994).

Planetary nebulae are good sites in which to probe the oxygen abundance, and they are the only sites that are directly accessible in DHGs. Since planetary nebulae are bright in strong emission lines (e.g., [O III] $]$ 5007), they are easily located within their parent galaxies using emission-line and continuum-band imaging (e.g., Ciardullo et al. 1989). Observational and theoretical evidence indicates that the stellar precursors of most planetary nebulae do not modify their initial oxygen abundance (Iben \& Renzini 1983; Henry 1989; Perinotto 1991; Forestini \& Charbonnel 1997). Hence, a planetary nebula's oxygen abundance reflects that in the interstellar medium at the time of its precursor's formation. Finally, most of the stellar populations in DHGs are old, so they will produce planetary nebulae at comparable rates per unit mass. As a result, planetary nebulae sample the oxygen abundances in DHGs according to the mass in each stellar population. The resulting mean oxygen abundance for the planetary nebula population in a DHG should then be a mass-weighted mean of the oxygen abundances in its stellar populations.
Apart from their utility for studying the chemical evolution of M 31 and M 32, the spectroscopic data for the planetary nebulae we present are interesting for what they reveal about the evolution of the planetary nebulae themselves. Though there may exist a good qualitative understanding of planetary nebula evolution, it is unclear how well it stands up to quantitative scrutiny. This situation arises primarily because the distances to planetary nebulae are difficult to establish within the Milky Way. Traditionally, this constraint has made it difficult to study such absolute properties as the luminosity and size of planetary nebulae, as well as the temporal evolution of these quantities. Extragalactic planetary nebulae are especially valuable in this regard because their distances are known. The addition of the data sets for M 32 and the bulge of M 31 is particularly helpful since these planetary nebulae arise from old stellar populations. They will thus provide an intriguing contrast with the planetary nebula populations in the Magellanic Clouds, which are the product of recent star formation (Richer 1993). Whether the evolution of planetary nebulae depends upon the progenitor mass or metallicity are among the questions that we may hope to answer through a comparison of the properties of planetary nebulae in M 31 and M 32 with those elsewhere. A better quantitative understanding of planetary nebula evolution would be a great help in understanding and using the planetary nebula luminosity function as a distance indicator.

In this paper, we present our spectroscopic data for our samples of planetary nebulae in M 32 and in the bulge of M 31. The observations and their reductions are described in Sect. 2. The line intensities and reddenings we deduce are presented in Sect. 3. The reddening-corrected line intensities are then used to calculate electron temperatures and oxygen abundances in Sect. 4. Summary comments are given in Sect. 5 .

In companion papers, we will use the data we present below to study the chemical evolution of DHGs and the evolution of planetary nebulae in different environments. 
Table 2. B600/O300 sensitivity correction

\begin{tabular}{lcl}
\hline Wavelength & $I(\mathrm{~B} 600) / I(\mathrm{O} 300)$ & M 32 Planetary Nebulae Used \\
\hline $\mathrm{H} \beta$ & $1.17 \pm 0.06$ & all, except PN25 \\
{$[\mathrm{O}$ III $] \lambda 4959$} & $1.00 \pm 0.01$ & $\mathrm{PN} 1, \mathrm{PN} 2, \mathrm{PN} 4, \mathrm{PN} 5, \mathrm{PN} 7, \mathrm{PN} 8, \mathrm{PN} 17, \mathrm{PN} 24$ \\
$\mathrm{He}$ I $\lambda 5876$ & $0.77 \pm 0.03$ & $\mathrm{PN} 1, \mathrm{PN} 7, \mathrm{PN} 8, \mathrm{PN} 11, \mathrm{PN} 17$ \\
$\mathrm{H} \alpha$ & $0.85 \pm 0.02$ & $\mathrm{PN} 2, \mathrm{PN} 8, \mathrm{PN} 11, \mathrm{PN} 17, \mathrm{PN} 24$ \\
& & \\
\hline
\end{tabular}

${ }^{\text {a }}$ PN4 and PN17 are background objects in the disk of M 31 (Ford \& Jenner 1975).

\section{Observations and reductions}

Our ultimate purpose for making these observations was to study the chemical evolution of M 32 and the bulge of M 31. In M 31, we chose planetary nebulae in the inner bulge in order to probe the highest levels of enrichment. On account of the bright galaxy background, we also preferentially chose planetary nebulae that were known to be bright in $[\mathrm{O}$ III $] \lambda 5007$. All of the objects we observed in M 31 are found within the inner half effective radius of M 31's bulge. In M 32, we observed as many objects as we could, again emphasizing bright objects on account of the galaxy background. In this case, the objects we observed extended to many effective radii.

We obtained our observations over three nights in August 1994 at the Canada-France-Hawaii Telescope (CFHT) with the multi-object spectrograph (MOS). The MOS is an imaging, multi-slit spectrograph that employs a grism as the dispersing element (see Le Fèvre et al. 1994 for details). Objects are selected for spectroscopy using focal plane masks that are constructed on-line from previously acquired images. The detector was the Loral3 CCD, a thick CCD with $15 \mu \mathrm{m}$ square pixels in a $2048 \times 2048$ format, coated to enhance the quantum efficiency in the blue. The Loral3's read noise was 8 electrons and its gain was set to 1.9 electrons/ADU. For the observations of both M 31 and M 32, we used slits $15^{\prime \prime}$ long by $1^{\prime \prime}$ wide. No order-sorting filter was used for any of these observations.

Table 1 presents a log of our observations. During the course of the observations, we used three different grism set-ups in order to optimize throughput, wavelength coverage, and spectral resolution. We used the B600 grism only because of the disappointing throughput of the U900 grism. Although the precise dispersion and wavelength coverage depend upon each object's position within the field of view, Table 1 lists typical values for all three grisms (minimal ranges for the wavelength coverage).

We used the standard IRAF routines to reduce the data (noao.imred.ccdred), and followed the standard reduction procedure. First, the overscan bias was removed from all of the images. Next, for the first two nights, sequences of zero exposure images were combined and subtracted from the other images to remove any bias pattern. This was not done on the third night because the CCD dewar began to warm up before we had a chance to obtain the zero exposure images. This is unlikely to be a limitation, since no bias pattern was obvious on either of the first two nights. Finally, pixel-to-pixel variations were removed using spectra of the internal quartz lamp.

Extracting the spectra proved challenging on account of the nature and faintness of the sources, and on account of the characteristics of the spectrograph. The planetary nebulae in M 31 and M 32 are sufficiently faint that we were unable to detect their continuum emission. Only the emission lines were visible, appearing as a sequence of dots, so it was impossible to trace these spectra. Furthermore, the spectra spanned the full width of the detector, so they suffered from geometric distortion (pin-cushion) introduced by the optics of the spectrograph. Fortunately, we had to include star apertures when defining the spectrograph's focal plane mask to permit accurate re-alignment on the field when ready to do spectroscopy. We used these stars ( 6 for M 31, 3 for M 32) to map the geometric distortion imposed by the optics, and corrected this distortion using the tasks in the noao.twodspec.longslit package (Anderson 1987). At this point, we had images in which the wavelength axis was parallel to the rows of the CCD, and we could use the brightest line in each spectrum to define an extraction aperture (e.g., Massey et al. 1992). Except for the U900 spectra, the individual spectra were extracted from each image and then combined to produce the final combined spectra. To better define the extraction apertures for the U900 spectra, the spectra were combined first, after verifying that the individual images had the same spatial coordinate scales. In all cases, extraction involved local subtraction of the underlying galaxy and sky spectra.

Establishing a consistent sensitivity scale across all three grism set-ups was a primary consideration of our data reduction. We calibrated the instrumental sensitivity for each set-up using observations of the spectrophotometric standard stars listed in Table 1 . We verified that our slitlet-to-slitlet sensitivity scale was secure in three ways. First, the observations of the standard stars were made in pairs through two different slitlets. These slitlets were cut at the red and blue extremes of the field of view to ensure that our standard star observations spanned the full wavelength range of our planetary nebula observations. These paired observations of the standard stars had $500 \AA$, $800 \AA$, and $1900 \AA$ of spectrum in common for the U900, $\mathrm{B} 600$, and $\mathrm{O} 300$ grisms, respectively. In these overlap regions, the sensitivity functions for each grism (on each 
night) were in agreement. Second, we obtained a spectroscopic sky flat through the standard star mask with the B600 grism on the last night. This mask contained two slitlets in addition to those used for the standard star observations. Comparing the night sky spectra through these four slitlets indicates that variations in the wavelength sensitivity between different slitlets are less than $4.5 \%$ (rms). Finally, observations of NGC 6720 were obtained through a different mask than the standard stars, and no wavelength-dependent trends are seen in its sensitivity calibration (see Table 3 below). Therefore, though we did not observe the standard stars through the slitlets used for our program objects, we have no reason to believe that our sensitivity calibration is slitlet-dependent.

We then chose the O300 observations of the planetary nebulae in M 32 as our reference data set. This choice was motivated by a number of considerations. First, these planetary nebulae were observed with all three grisms. Second, the $\mathrm{O} 300$ grism has good sensitivity over the $\mathrm{H} \beta$ - H $\alpha$ wavelength range (Le Fèvre et al. 1994), which contains the strongest lines in the spectra. Third, our reddening values for these planetary nebulae (see Tables 6 , 7 , and 8 ) were reasonable, typically $E(B-V)<0.2 \mathrm{mag}$, and invariably positive. These reddenings were consistent with previous observations of PN1 in M 32 (Ford et al. 1978). The reddening towards M 32 is also expected to be small if it is in front of the disk of M 31 (e.g., Burstein \& Heiles 1984).

We ensured that there were no systematic differences between the $\mathrm{B} 600$ and $\mathrm{O} 300$ data sets by comparing the intensities of $\mathrm{H} \beta, \mathrm{H} \alpha$, [O III $] \lambda 4959$, and $\mathrm{He}$ I $\lambda 5876$ measured relative to $[\mathrm{O}$ III $] \lambda 5007$ for the planetary nebulae in M 32. In making these comparisons, we considered only those objects for which we had the best detections of these lines. For these objects, we computed the ratio of the line intensity in the B600 spectrum to that in the O300 spectrum. Table 2 lists the mean value of this ratio, the standard error in the mean, and the objects we considered for each line. Clearly, the main wavelength-dependent trend in Table 2 is a systematic decrease in the B600 sensitivity relative to the $\mathrm{O} 300$ sensitivity as one goes to longer wavelengths. Simply fitting a line to the values in Table 2 as a function of wavelength, however, yields a rather poor correction at $\mathrm{H} \alpha$. As a result, for wavelengths between any two lines found in Table 2, we corrected for the difference in sensitivity calibrations by interpolating linearly between the corrections in Table 2. For lines to the blue of $\mathrm{H} \beta$ or to the red of $\mathrm{H} \alpha$, we adopted the $\mathrm{H} \beta$ or $\mathrm{H} \alpha$ corrections, respectively. We wondered if the upturn at $\mathrm{H} \alpha$ in Table 2 could be due to second order contamination, but this seems unlikely. Both the O300 and B600 grisms have very low efficiency at $3250 \AA$, and a second order contamination would affect the sensitivity calibration for both grisms similarly. Consequently, the upturn at $\mathrm{H} \alpha$ appears to be real. The corrections in Table 2 were applied
Table 3. Hydrogen line intensities for NGC 6720

\begin{tabular}{|c|c|c|c|}
\hline & Line & Intensity $^{\mathrm{a}}$ & $E(B-V)^{\mathrm{a}}$ \\
\hline \multirow[t]{9}{*}{ Aperture 1} & $\mathrm{H} \beta$ & $100.00 \pm 1.49$ & \\
\hline & $\mathrm{H} \gamma$ & $42.07 \pm 0.55$ & $0.287 \pm 0.034$ \\
\hline & $\mathrm{H} \delta$ & $24.03 \pm 0.33$ & $0.133 \pm 0.023$ \\
\hline & $\mathrm{H} \epsilon$ & $12.99 \pm 0.87$ & $0.279 \pm 0.093$ \\
\hline & $\mathrm{H} 8$ & $7.14 \pm 0.35$ & $0.484 \pm 0.061$ \\
\hline & H9 & $5.79 \pm 0.13$ & $0.280 \pm 0.027$ \\
\hline & H10 & $3.97 \pm 0.13$ & $0.331 \pm 0.037$ \\
\hline & H11 & $2.85 \pm 0.16$ & $0.371 \pm 0.060$ \\
\hline & H12 & $2.32 \pm 0.15$ & $0.304 \pm 0.070$ \\
\hline \multirow[t]{9}{*}{ Aperture 2} & $\mathrm{H} \beta$ & $100.00 \pm 1.53$ & \\
\hline & $\mathrm{H} \gamma$ & $42.29 \pm 0.57$ & $0.273 \pm 0.035$ \\
\hline & $\mathrm{H} \delta$ & $24.06 \pm 0.34$ & $0.131 \pm 0.024$ \\
\hline & $\mathrm{H} \epsilon$ & $13.13 \pm 0.90$ & $0.264 \pm 0.094$ \\
\hline & H8 & $7.24 \pm 0.36$ & $0.467 \pm 0.063$ \\
\hline & H9 & $5.59 \pm 0.19$ & $0.320 \pm 0.041$ \\
\hline & H10 & $3.89 \pm 0.15$ & $0.355 \pm 0.043$ \\
\hline & H11 & $2.84 \pm 0.19$ & $0.375 \pm 0.073$ \\
\hline & $\mathrm{H} 12$ & $2.19 \pm 0.18$ & $0.366 \pm 0.089$ \\
\hline \multirow[t]{9}{*}{ Aperture 3} & $\mathrm{H} \beta$ & $100.00 \pm 1.45$ & \\
\hline & $\mathrm{H} \gamma$ & $42.51 \pm 0.56$ & $0.259 \pm 0.035$ \\
\hline & $\mathrm{H} \delta$ & $24.72 \pm 0.37$ & $0.085 \pm 0.025$ \\
\hline & $\mathrm{H} \epsilon$ & $13.11 \pm 0.84$ & $0.265 \pm 0.088$ \\
\hline & H8 & $7.91 \pm 0.43$ & $0.355 \pm 0.068$ \\
\hline & H9 & $5.58 \pm 0.26$ & $0.323 \pm 0.056$ \\
\hline & H10 & $3.77 \pm 0.22$ & $0.391 \pm 0.064$ \\
\hline & H11 & $3.15 \pm 0.37$ & $0.262 \pm 0.129$ \\
\hline & H12 & $2.60 \pm 0.37$ & $0.184 \pm 0.151$ \\
\hline
\end{tabular}

a The derivation of the uncertainties in the line intensities and reddening is described in Sect. 3.

to the spectra of the planetary nebulae in both M 32 and the bulge of M 31 .

The U900 data required no correction to put them on the O300 sensitivity scale. We deduced this from direct comparison with the B600 and O300 data (Tables 6, 7, and 8), and independently using a spectrum we obtained of the Galactic planetary nebula NGC 6720. Table 3 lists the intensities and reddening values for hydrogen lines in three regions of NGC 6720 . The reddening values we derive from $\mathrm{H} \gamma, \mathrm{H} \epsilon, \mathrm{H} 9, \mathrm{H} 10, \mathrm{H} 11$, and $\mathrm{H} 12$ are in very good agreement in all three apertures, indicating that our U900 sensitivity calibration is good to $3750 \AA$. Our reddening values at $\mathrm{H} \delta$ are consistently $0.16 \mathrm{mag}$ lower than calculated from $\mathrm{H} \gamma$, so our U900 sensitivities may be underestimated by $15 \%$ near $4100 \AA$. Our H8 reddening values are consistently high, but $\mathrm{H} 8$ was blended with He I $\lambda 3889$. We corrected the blend for the He I $\lambda 3889$ contribution using the He I $\lambda 4471$ intensity assuming no radiative transfer correction, thereby removing the maximum possible He I $\lambda 3889$ contribution (e.g., Aller 1987). Thus, it is perhaps not surprising that our $\mathrm{H} 8$ reddenings are too high. Overall, our Balmer line intensities for NGC 6720 indicate that our U900 sensitivity calibration is secure from $3750 \AA$ to $\mathrm{H} \beta$. Similarly, for the planetary nebulae in 
Table 4. Oxygen abundances in M 32

\begin{tabular}{llll}
\hline Object & $T_{\mathrm{e}}$ & $\begin{array}{l}12+\log (\mathrm{O} / \mathrm{H}) \\
\text { no ICF } \\
(\text { dex })\end{array}$ & $\begin{array}{l}12+\log (\mathrm{O} / \mathrm{H}) \\
\text { KB94 } \mathrm{ICF}^{\mathrm{a}} \\
(\text { dex}, \text { adopted })\end{array}$ \\
\hline PN8 & $1 \mathrm{~K})$ & $8.36 \pm 0.14$ & $8.38 \pm 0.14$ \\
PN11 & $<25300$ & $>7.25$ & $>7.31$ \\
PN2 & $<15700$ & $>8.17$ & $>8.27$ \\
PN7 & $<13400$ & $>8.08$ & $>8.10$ \\
PN25 & $<18600$ & $>8.02$ & $>8.08$ \\
PN24 & $18200 \pm 2500$ & $8.07 \pm 0.13$ & $8.13 \pm 0.13$ \\
PN6 & $12700 \pm 2500$ & $8.46 \pm 0.27$ & $8.50 \pm 0.27$ \\
PN5 & $11800 \pm 1300$ & $8.43 \pm 0.16$ & $8.46 \pm 0.16$ \\
PN1 & $<11500$ & $>8.39$ & $>8.41$ \\
PN4 & $<12200$ & $>8.58$ & $>8.72$ \\
PN17 & $<18400$ & $>7.84$ & $>7.92$ \\
\hline
\end{tabular}

a Kingsburgh \& Barlow 1994 ICF.

b PN4 and PN17 are background objects in the disk of M 31 (Ford \& Jenner 1975).

M 32 (Tables 6, 7, and 8), the U900 line intensities for [O II] $\lambda 3727$, [Ne III] $\lambda 3869$, and He II $\lambda 4686$ are in excellent agreement with their B600 and O300 counterparts.

Figures 1 through 6 display the O300, B600, and U900 spectra of the planetary nebulae in M 32, while Figs. 7 through 12 display the B600 spectra of the planetary nebulae in the bulge of M 31. The object designations (Ciardullo et al. 1989) are shown next to the spectra. Normally, the spectra are scaled such that $\mathrm{H} \beta$ occupies the full intensity scale, so stronger lines from adjacent spectra overlap, but some of the U900 and B600 spectra are scaled such that $\mathrm{H} \gamma$ and $\mathrm{H} \alpha$, respectively, occupy the full intensity scale. This scaling allowed the best compromise in demonstrating the signal-to-noise for various lines and an assessment of the background sky and galaxy subtraction. The full wavelength range is shown for the B600 and U900 spectra, but only the wavelength range below $7350 \AA$ is shown for the $\mathrm{O} 300$ spectra. Cosmic rays were not removed unless they interfered with the measurement of line intensities, and many remain in the spectra displayed in Figs. 1 through 12 .

\section{Line intensities and reddening}

Tables 6 to 9 (at end) list the adopted reddening-corrected line intensity ratios and reddening values for the planetary nebulae in M 32 and the bulge of M 31. We use the object designations from Ciardullo et al. (1989). The line intensities were measured using the software described by McCall et al. (1985). The uncertainties quoted for the line ratios are $1 \sigma$ uncertainties that incorporate the uncertainties in both the line and $\mathrm{H} \beta$ fluxes. The uncertainties in the line fluxes include contributions from the fit to the line itself and from the noise in the continuum. In those instances where there is no line intensity value, but there is a line
Table 5. Oxygen abundances in M 31

\begin{tabular}{|c|c|c|c|}
\hline Object & $\begin{array}{l}T_{\mathrm{e}} \\
(\mathrm{K})\end{array}$ & $\begin{array}{l}12+\log (\mathrm{O} / \mathrm{H}) \\
\text { no ICF } \\
(\mathrm{dex})\end{array}$ & $\begin{array}{l}12+\log (\mathrm{O} / \mathrm{H}) \\
\mathrm{KB} 94 \mathrm{ICF}^{\mathrm{a}} \\
(\text { dex, adopted })\end{array}$ \\
\hline PN172 & $13100 \pm 1100$ & $8.44 \pm 0.10$ & $8.46 \pm 0.10$ \\
\hline PN31 & $10700 \pm 780$ & $8.80 \pm 0.10$ & $8.80 \pm 0.10$ \\
\hline PN80 & $<9500$ & $>8.91$ & $>8.91$ \\
\hline PN30 & $<9300$ & $>9.00$ & $>9.00$ \\
\hline PN29 & $<9800$ & $>8.91$ & $>8.91$ \\
\hline PN28 & $<8600$ & $>9.11$ & $>9.11$ \\
\hline $\mathrm{PN} 23$ & $<13200$ & $>8.30$ & $>8.30$ \\
\hline PN12 & $13100 \pm 2000$ & $8.44 \pm 0.18$ & $8.51 \pm 0.18$ \\
\hline PN10 & $<13600$ & $>8.22$ & $>8.24$ \\
\hline PN1 & $<10100$ & $>8.79$ & $>8.79$ \\
\hline PN3 & $<9400$ & $>8.96$ & $>8.96$ \\
\hline PN38 & $<10500$ & $>8.58$ & $>8.58$ \\
\hline PN36 & $14600 \pm 1600$ & $8.12 \pm 0.12$ & $8.15 \pm 0.12$ \\
\hline PN53 & $9600 \pm 870$ & $8.91 \pm 0.14$ & $8.92 \pm 0.14$ \\
\hline PN52 & $<11300$ & $>8.53$ & $>8.64$ \\
\hline PN42 & $12400 \pm 750$ & $8.57 \pm 0.08$ & $8.59 \pm 0.08$ \\
\hline PN45 & $15100 \pm 940$ & $8.16 \pm 0.07$ & $8.17 \pm 0.07$ \\
\hline PN43 & $<10200$ & $>8.71$ & $>8.72$ \\
\hline PN48 & $9500 \pm 640$ & $8.95 \pm 0.11$ & $8.96 \pm 0.11$ \\
\hline PN95 & $<9300$ & $>8.66$ & $>8.66$ \\
\hline PN47 & $9600 \pm 1200$ & $8.77 \pm 0.20$ & $8.78 \pm 0.20$ \\
\hline PN408 & $<13000$ & $>8.52$ & $>8.52$ \\
\hline PN93 & $<10500$ & $>8.79$ & $>8.80$ \\
\hline PN92 & $11800 \pm 1100$ & $8.56 \pm 0.12$ & $8.57 \pm 0.12$ \\
\hline PN97 & $9400 \pm 1000$ & $8.87 \pm 0.18$ & $8.88 \pm 0.18$ \\
\hline PN91 & $12700 \pm 1400$ & $8.38 \pm 0.13$ & $8.38 \pm 0.13$ \\
\hline PN387 & $10600 \pm 810$ & $8.71 \pm 0.12$ & $8.74 \pm 0.12$ \\
\hline PN380 & $13900 \pm 1100$ & $8.38 \pm 0.09$ & $8.39 \pm 0.09$ \\
\hline
\end{tabular}

${ }^{\text {a }}$ Kingsburgh \& Barlow 1994 ICF.

intensity uncertainty, e.g., He II $\lambda 4686$ in PN5 in M 32, the "uncertainty" is a $2 \sigma$ upper limit to the strength of undetected lines, and is based upon the noise observed in the continuum. Note that PN4 and PN17 in the M 32 field have radial velocities indicating that they belong to the background disk of M 31 (Ford \& Jenner 1975). The H II region in the background disk of M 31 that we observed in the M 32 field is that denoted H II 1 by Ford \& Jenner (1975).

For the planetary nebulae in M 32, Tables 6 to 8 list the reddening-corrected O300, B600, and U900 line intensities, in addition to our adopted line intensities. The adopted intensities are those listed under the object name. Generally, we adopted the U900 line intensities in the blue and the $\mathrm{O} 300$ line intensities in the red, with the dividing line being He II $\lambda 4686$. He II $\lambda 4686$ is the only common exception to this rule. For He II $\lambda 4686$, we normally chose the line intensity from the spectrum in which the line was measured with the lowest relative error. 
Table 6. Line intensities for PNe in M 32

\begin{tabular}{|c|c|c|c|c|c|c|c|c|}
\hline Wavelength & PN5 & O300 & $\mathrm{B} 600$ & U900 & PN1 & O300 & B600 & U900 \\
\hline $3727^{\mathrm{a}}$ & $29.0 \pm 8.3$ & & & $29.0 \pm 8.3$ & $83 \pm 13$ & & & $83 \pm 13$ \\
\hline 3868.76 & $94.0 \pm 9.9$ & & $100 \pm 19$ & $94.0 \pm 9.9$ & $110 \pm 12$ & & $78 \pm 13$ & $110 \pm 12$ \\
\hline 3967.47 & $48.6 \pm 9.3$ & & & $48.6 \pm 9.3$ & $49 \pm 11$ & & & $49 \pm 11$ \\
\hline 4101.737 & $29.8 \pm 5.8$ & & & $29.8 \pm 5.8$ & $33.3 \pm 5.8$ & & $27.1 \pm 8.5$ & $33.3 \pm 5.8$ \\
\hline 4340.468 & $47.0 \pm 6.6$ & & \pm 13 & $47.0 \pm 6.6$ & $47.0 \pm 6.8$ & $48.6 \pm 8.8$ & $42.2 \pm 5.9$ & $47.0 \pm 6.8$ \\
\hline 4363.21 & $14.0 \pm 4.4$ & & \pm 13 & $14.0 \pm 4.4$ & \pm 10 & \pm 16 & \pm 9 & \pm 10 \\
\hline 4685.682 & \pm 17 & \pm 17 & \pm 14 & $10.0 \pm 3.3$ & $10.8 \pm 3.7$ & \pm 7 & $10.8 \pm 3.7$ & \pm 11 \\
\hline 4861.332 & $100.0 \pm 5.4$ & $100.0 \pm 5.4$ & $100.0 \pm 8.8$ & & $100.0 \pm 4.0$ & $100.0 \pm 4.0$ & $100.0 \pm 4.8$ & \\
\hline 4958.92 & $431.7 \pm 5.0$ & $431.7 \pm 5.0$ & $448 \pm 26$ & & $337.8 \pm 4.6$ & $337.8 \pm 4.6$ & $349 \pm 11$ & \\
\hline 5006.85 & $1291.8 \pm 6.9$ & $1291.8 \pm 6.9$ & $1343 \pm 71$ & & $994.1 \pm 6.7$ & $994.1 \pm 6.7$ & $1052 \pm 29$ & \\
\hline 5875.666 & $16.0 \pm 4.0$ & $16.0 \pm 4.0$ & $18.8 \pm 4.9$ & & $21.2 \pm 1.6$ & $21.2 \pm 1.6$ & $22.3 \pm 2.6$ & \\
\hline 6300.32 & \pm 6 & \pm 6 & & & \pm 2 & \pm 2 & & \\
\hline 6312.06 & $10.4 \pm 3.6$ & $10.4 \pm 3.6$ & & & \pm 2 & \pm 2 & & \\
\hline 6363.81 & \pm 6 & \pm 6 & & & \pm 2 & \pm 2 & & \\
\hline 6548.06 & $29.6 \pm 3.2$ & $29.6 \pm 3.2$ & & & $76.7 \pm 2.1$ & $76.7 \pm 2.1$ & & \\
\hline 6562.817 & $285.0 \pm 4.0$ & $285.0 \pm 4.0$ & & & $285.0 \pm 2.7$ & $285.0 \pm 2.7$ & & \\
\hline 6583.39 & $102.7 \pm 3.5$ & $102.7 \pm 3.5$ & & & $230.6 \pm 2.5$ & $230.6 \pm 2.5$ & & \\
\hline 6716.42 & & & & & $10.21 \pm 0.92$ & $10.21 \pm 0.92$ & & \\
\hline 6730.72 & & & & & $17.08 \pm 0.99$ & $17.08 \pm 0.99$ & & \\
\hline 7065.277 & $13.0 \pm 4.1$ & $13.0 \pm 4.1$ & & & $3.5 \pm 1.0$ & $3.5 \pm 1.0$ & & \\
\hline 7135.8 & $30.2 \pm 4.8$ & $30.2 \pm 4.8$ & & & $18.0 \pm 1.3$ & $18.0 \pm 1.3$ & & \\
\hline 7325 & $10.4 \pm 3.3$ & $10.4 \pm 3.3$ & & & $3.11 \pm 0.56$ & $3.11 \pm 0.56$ & & \\
\hline 9069 & $12.7 \pm 3.1$ & $12.7 \pm 3.1$ & & & & & & \\
\hline$E(B-V)$ & $0.01 \pm 0.01$ & & & & $0.11 \pm 0.01$ & & & \\
\hline Wavelength & PN4, M 31 & O300 & $\mathrm{B} 600$ & U900 & PN17, M 31 & O300 & B600 & U900 \\
\hline $3727^{\mathrm{a}}$ & $106 \pm 36$ & & & $106 \pm 36$ & \pm 64 & & & \pm 64 \\
\hline 3868.76 & $169 \pm 32$ & & & $169 \pm 32$ & $79 \pm 22$ & & & $79 \pm 22$ \\
\hline 3967.47 & $53 \pm 15$ & & & $53 \pm 15$ & & & & \\
\hline 4101.737 & $50 \pm 17$ & & & $50 \pm 17$ & \pm 34 & & & \pm 34 \\
\hline 4340.468 & $47 \pm 13$ & & & $47 \pm 13$ & $41 \pm 13$ & & & $41 \pm 13$ \\
\hline 4363.21 & \pm 22 & & & \pm 22 & \pm 29 & & & \pm 29 \\
\hline 4685.682 & $51 \pm 11$ & $51 \pm 11$ & $37 \pm 10$ & $55 \pm 14$ & \pm 22 & \pm 26 & \pm 25 & \pm 22 \\
\hline 4861.332 & $100 \pm 11$ & $100 \pm 11$ & $100 \pm 12$ & & $100 \pm 12$ & $100 \pm 12$ & $100 \pm 14$ & $100 \pm 10$ \\
\hline 4958.92 & $637 \pm 10$ & $637 \pm 10$ & $703 \pm 50$ & & $337 \pm 12$ & $337 \pm 12$ & $384 \pm 38$ & $253 \pm 18$ \\
\hline 5006.85 & $1916 \pm 14$ & $1916 \pm 14$ & $2137 \pm 149$ & & $993 \pm 16$ & $993 \pm 16$ & $1176 \pm 110$ & $807 \pm 48$ \\
\hline 5875.666 & $10.9 \pm 4.7$ & \pm 4 & $10.9 \pm 4.7$ & & $13.0 \pm 3.4$ & $13.0 \pm 3.4$ & $19.3 \pm 4.9$ & \\
\hline 6300.32 & \pm 6 & \pm 6 & & & \pm 4 & \pm 4 & & \\
\hline 6312.06 & \pm 6 & \pm 6 & & & \pm 4 & \pm 4 & & \\
\hline 6363.81 & \pm 6 & \pm 6 & & & \pm 4 & \pm 4 & & \\
\hline 6548.06 & $15.6 \pm 4.0$ & $15.6 \pm 4.0$ & & & $26.3 \pm 2.4$ & $26.3 \pm 2.4$ & $29.6 \pm 5.3$ & \\
\hline 6562.817 & $285.0 \pm 5.7$ & $285.0 \pm 5.7$ & & & $285.0 \pm 4.1$ & $285.0 \pm 4.1$ & $349 \pm 33$ & \\
\hline 6583.39 & $45.4 \pm 4.5$ & $45.4 \pm 4.5$ & & & $77.3 \pm 2.7$ & $77.3 \pm 2.7$ & $91.7 \pm 9.8$ & \\
\hline 7135.8 & $21.8 \pm 3.1$ & $21.8 \pm 3.1$ & & & $22.5 \pm 4.1$ & & $22.5 \pm 4.1$ & \\
\hline 7325 & $14.4 \pm 5.4$ & $14.4 \pm 5.4$ & & & $14.2 \pm 1.9$ & $14.2 \pm 1.9$ & & \\
\hline$E(B-V)$ & $0.15 \pm 0.02$ & & & & $0.57 \pm 0.01$ & & & \\
\hline
\end{tabular}

a " 3727 " denotes the sum of $[\mathrm{O} \mathrm{II}] \lambda \lambda 3726,3729$.

The reddening-corrected line intensities in Tables 6 through 9 are related to those we observed via

$\log \frac{I(\lambda)}{I(\mathrm{H} \beta)}=\log \frac{F(\lambda)}{F(\mathrm{H} \beta)}-0.4 E(B-V)(A(\lambda)-A(\mathrm{H} \beta))$

where $F(\lambda)$ and $I(\lambda)$ are the observed and reddeningcorrected line intensities, respectively, $E(B-V)$ is the reddening, and $A(\lambda)$ is the extinction for $E(B-V)=1.0 \mathrm{mag}$ from the reddening law of Schild (1977). All of the line intensities for the planetary nebulae in M 32 in Tables 6, 7, and 8 have been corrected for reddening using $E(B-V)$ determined from the $\mathrm{O} 300 \mathrm{H} \alpha / \mathrm{H} \beta$ ratio. For the U900 spectra that did not extend to $\mathrm{H} \beta$, we corrected intensities relative to $\mathrm{H} \gamma$ using the $\mathrm{O} 300$ reddening, then adopted $I(\mathrm{H} \gamma) / I(\mathrm{H} \beta)=0.47$. For the planetary nebulae in the bulge of $\mathrm{M} 31$, we determined the reddening from the 
Table 7. Line intensities for PNe in M 32 (continued)

\begin{tabular}{|c|c|c|c|c|c|c|c|c|}
\hline Wavelength & PN8 & O300 & $\mathrm{B} 600$ & U900 & PN2 & O300 & B600 & U900 \\
\hline 3726.05 & $38.1 \pm 9.0$ & & & $38.1 \pm 9.0$ & \pm 35 & & & \pm 35 \\
\hline 3728.8 & $41.9 \pm 9.1$ & & & $41.9 \pm 9.1$ & $85 \pm 22$ & & & $85 \pm 22$ \\
\hline 3868.76 & $121 \pm 10$ & & & $121 \pm 10$ & $98 \pm 17$ & & & $98 \pm 17$ \\
\hline 3967.47 & $62 \pm 13$ & & & $62 \pm 13$ & $60 \pm 17$ & & & $60 \pm 17$ \\
\hline 4101.737 & $24.1 \pm 7.6$ & & & $24.1 \pm 7.6$ & \pm 21 & & & \pm 21 \\
\hline 4340.468 & $59.7 \pm 6.8$ & & $42.4 \pm 6.0$ & $59.7 \pm 6.8$ & $73 \pm 17$ & & & $73 \pm 17$ \\
\hline 4363.21 & $19.1 \pm 5.3$ & & $13.4 \pm 4.7$ & $19.1 \pm 5.3$ & \pm 31 & & & \pm 31 \\
\hline 4685.682 & \pm 11 & $5.3 \pm 2.5$ & \pm 8 & \pm 11 & $22.8 \pm 7.7$ & $16.5 \pm 7.9$ & $44 \pm 11$ & $22.8 \pm 7.7$ \\
\hline 4861.332 & $100.0 \pm 4.6$ & $100.0 \pm 4.6$ & $100.0 \pm 6.0$ & $100.0 \pm 7.1$ & $100 \pm 10$ & $100 \pm 10$ & $100 \pm 13$ & $100 \pm 11$ \\
\hline 4958.92 & $462.5 \pm 7.6$ & $462.5 \pm 7.6$ & $458 \pm 17$ & $434 \pm 18$ & $508.7 \pm 7.3$ & $508.7 \pm 7.3$ & $723 \pm 61$ & $520 \pm 36$ \\
\hline 5006.85 & $1386 \pm 11$ & $1386 \pm 11$ & $1364 \pm 48$ & $1334 \pm 52$ & $1461.1 \pm 9.5$ & $1461.1 \pm 9.5$ & $2109 \pm 172$ & $1602 \pm 100$ \\
\hline 5875.666 & $16.6 \pm 1.1$ & $16.6 \pm 1.1$ & $18.2 \pm 2.2$ & & $8.3 \pm 2.5$ & $8.3 \pm 2.5$ & $16.4 \pm 5.6$ & \\
\hline 6300.32 & $5.8 \pm 1.4$ & $5.8 \pm 1.4$ & & & $8.6 \pm 2.6$ & $8.6 \pm 2.6$ & & \\
\hline 6312.06 & $3.2 \pm 1.3$ & $3.2 \pm 1.3$ & & & \pm 3 & \pm 3 & & \\
\hline 6363.81 & \pm 2 & \pm 2 & & & \pm 3 & \pm 3 & & \\
\hline 6548.06 & $57.0 \pm 1.2$ & $57.0 \pm 1.2$ & $51.0 \pm 3.7$ & & $30.9 \pm 2.0$ & $30.9 \pm 2.0$ & $50.6 \pm 8.3$ & \\
\hline 6562.817 & $285.0 \pm 1.9$ & $285.0 \pm 1.9$ & $275.4 \pm 10$ & & $285.0 \pm 2.7$ & $285.0 \pm 2.7$ & $414 \pm 35$ & \\
\hline 6583.39 & $168.2 \pm 1.5$ & $168.2 \pm 1.5$ & $158.2 \pm 6.7$ & & $102.4 \pm 2.3$ & $102.4 \pm 2.3$ & $159 \pm 15$ & \\
\hline 6716.42 & $11.2 \pm 1.1$ & $11.2 \pm 1.1$ & $9.8 \pm 1.9$ & & $9.0 \pm 1.8$ & $9.0 \pm 1.8$ & \pm 13 & \\
\hline 6730.72 & $17.1 \pm 1.2$ & $17.1 \pm 1.2$ & & & $14.0 \pm 1.9$ & $14.0 \pm 1.9$ & & \\
\hline 7065.277 & $5.75 \pm 0.71$ & $5.75 \pm 0.71$ & & & & & & \\
\hline 7135.8 & $17.66 \pm 0.84$ & $17.66 \pm 0.84$ & $15.3 \pm 2.7$ & & $19.7 \pm 2.0$ & $19.7 \pm 2.0$ & $21.2 \pm 6.0$ & \\
\hline 7325 & $2.36 \pm 0.57$ & $2.36 \pm 0.57$ & & & & & & \\
\hline 9069 & $13.2 \pm 1.2$ & $13.2 \pm 1.2$ & & & $20.1 \pm 3.3$ & $20.1 \pm 3.3$ & & \\
\hline 9531.8 & $34.0 \pm 3.7$ & $34.0 \pm 3.7$ & & & $32.2 \pm 4.7$ & $32.2 \pm 4.7$ & & \\
\hline$E(B-V)$ & $0.20 \pm 0.01$ & & & & $0.06 \pm 0.01$ & & & \\
\hline Wavelength & PN11 & O300 & B600 & U900 & PN7 & O300 & B600 & U900 \\
\hline 3726.05 & $123 \pm 20$ & & & $123 \pm 20$ & $25.2 \pm 8.2$ & & & $25.2 \pm 8.2$ \\
\hline 3728.8 & $119 \pm 20$ & & & $119 \pm 20$ & \pm 11 & & & \pm 11 \\
\hline 3868.76 & $21.0 \pm 8.3$ & & & $21.0 \pm 8.3$ & $48 \pm 10$ & & $49 \pm 17$ & $48 \pm 10$ \\
\hline 3967.47 & $16.5 \pm 6.8$ & & & $16.5 \pm 6.8$ & $21.3 \pm 5.4$ & & & $21.3 \pm 5.4$ \\
\hline 4101.737 & $16.9 \pm 8.3$ & & & $16.9 \pm 8.3$ & $24.8 \pm 8.7$ & & & $24.8 \pm 8.7$ \\
\hline 4340.468 & $21.1 \pm 9.4$ & & \pm 47 & $21.1 \pm 9.4$ & $47.0 \pm 8.4$ & & $38.5 \pm 6.8$ & $47.0 \pm 8.4$ \\
\hline 4363.21 & \pm 14 & & & \pm 14 & \pm 12 & & \pm 9 & \pm 12 \\
\hline 4685.682 & \pm 16 & \pm 15 & \pm 21 & \pm 16 & $11.9 \pm 4.8$ & $11.9 \pm 4.8$ & \pm 9 & \\
\hline 4861.332 & $100.0 \pm 9.2$ & $100.0 \pm 9.2$ & $100 \pm 16$ & $100.0 \pm 9.5$ & $100.0 \pm 5.5$ & $100.0 \pm 5.5$ & $100.0 \pm 5.9$ & \\
\hline 4958.92 & $91.4 \pm 6.5$ & $91.4 \pm 6.5$ & $106 \pm 14$ & $94.6 \pm 9.2$ & $274.5 \pm 4.3$ & $274.5 \pm 4.3$ & $282 \pm 11$ & \\
\hline 5006.85 & $298.4 \pm 8.7$ & $298.4 \pm 8.7$ & $320 \pm 34$ & $263 \pm 17$ & $805.8 \pm 5.9$ & $805.8 \pm 5.9$ & $863 \pm 31$ & \\
\hline 5875.666 & $17.1 \pm 3.0$ & $17.1 \pm 3.0$ & $15.2 \pm 6.9$ & & $14.0 \pm 1.4$ & $14.0 \pm 1.4$ & $12.5 \pm 2.0$ & \\
\hline 6300.32 & \pm 4 & \pm 4 & & & & & & \\
\hline 6312.06 & \pm 4 & \pm 4 & & & & & & \\
\hline 6363.81 & \pm 4 & \pm 4 & & & & & & \\
\hline 6548.06 & $60.3 \pm 2.5$ & $60.3 \pm 2.5$ & $75.5 \pm 12.2$ & & $5.7 \pm 1.2$ & $5.7 \pm 1.2$ & & \\
\hline 6562.817 & $285.0 \pm 5.1$ & $285.0 \pm 5.1$ & $315 \pm 33$ & & $285.0 \pm 2.1$ & $285.0 \pm 2.1$ & & \\
\hline 6583.39 & $178.7 \pm 3.7$ & $178.7 \pm 3.7$ & $199 \pm 23$ & & $12.3 \pm 1.3$ & $12.3 \pm 1.3$ & & \\
\hline 6716.42 & $12.0 \pm 2.0$ & $12.0 \pm 2.0$ & \pm 13 & & \pm 3 & \pm 3 & & \\
\hline 6730.72 & $16.3 \pm 2.1$ & $16.3 \pm 2.1$ & & & \pm 3 & \pm 3 & & \\
\hline 7065.277 & & & & & $6.4 \pm 1.1$ & $6.4 \pm 1.1$ & & \\
\hline 7135.8 & $8.5 \pm 2.2$ & $8.5 \pm 2.2$ & & & $5.5 \pm 1.0$ & $5.5 \pm 1.0$ & & \\
\hline 7325 & $5.7 \pm 2.0$ & $5.7 \pm 2.0$ & & & & & & \\
\hline 9069 & $9.3 \pm 3.1$ & $9.3 \pm 3.1$ & & & & & & \\
\hline$E(B-V)$ & $0.05 \pm 0.02$ & & & & $0.03 \pm 0.01$ & & & \\
\hline
\end{tabular}


Table 8. Line intensities for PNe in M 32 (continued)

\begin{tabular}{|c|c|c|c|c|c|c|c|c|}
\hline Wavelength & H II \#1 & O300 & $\mathrm{B} 600$ & U900 & PN24 & O300 & $\mathrm{B} 600$ & U900 \\
\hline 3726.05 & $98 \pm 33$ & & & $98 \pm 33$ & $73 \pm 15$ & & & $73 \pm 15$ \\
\hline $3727^{\mathrm{a}}$ & & & $148 \pm 40$ & & & & & \\
\hline 3728.8 & $58 \pm 27$ & & & $58 \pm 27$ & $48 \pm 13$ & & & $48 \pm 13$ \\
\hline 3868.76 & $26 \pm 14$ & & & $26 \pm 14$ & $134 \pm 20$ & & $135 \pm 36$ & $134 \pm 20$ \\
\hline 3967.47 & & & & & $54 \pm 15$ & & & $54 \pm 15$ \\
\hline 4101.737 & \pm 21 & & & \pm 21 & \pm 30 & & & \pm 30 \\
\hline 4340.468 & $47 \pm 16$ & & \pm 13 & $47 \pm 16$ & $67 \pm 13$ & & $46 \pm 16$ & $67 \pm 13$ \\
\hline 4363.21 & \pm 23 & & \pm 13 & \pm 23 & $47 \pm 12$ & & & $47 \pm 12$ \\
\hline 4685.682 & \pm 17 & \pm 8 & \pm 17 & & $39 \pm 12$ & \pm 34 & $32 \pm 13$ & $39 \pm 12$ \\
\hline 4861.332 & $100.0 \pm 6.5$ & $100.0 \pm 6.5$ & $100 \pm 11$ & & $100 \pm 19$ & $100 \pm 19$ & $100 \pm 21$ & $100 \pm 14$ \\
\hline 4958.92 & $63.0 \pm 5.8$ & $63.0 \pm 5.8$ & $73.1 \pm 7.5$ & & $572 \pm 23$ & $572 \pm 23$ & $541 \pm 71$ & $465 \pm 41$ \\
\hline 5006.85 & $205.5 \pm 7.5$ & $205.5 \pm 7.5$ & $215 \pm 15$ & & $1654 \pm 30$ & $1654 \pm 30$ & $1585 \pm 202$ & \\
\hline 5875.666 & $11.5 \pm 1.8$ & $11.5 \pm 1.8$ & & & $22 \pm 10$ & $64 \pm 14$ & $22 \pm 10$ & \\
\hline 6300.32 & \pm 4 & \pm 4 & & & \pm 27 & \pm 27 & & \\
\hline 6312.06 & \pm 4 & \pm 4 & & & \pm 27 & \pm 27 & & \\
\hline 6363.81 & \pm 4 & \pm 4 & & & \pm 27 & \pm 27 & & \\
\hline 6548.06 & $28.3 \pm 2.3$ & $28.3 \pm 2.3$ & & & $94.7 \pm 9.7$ & $94.7 \pm 9.7$ & $63.0 \pm 15.1$ & \\
\hline 6562.817 & $285.0 \pm 5.5$ & $285.0 \pm 5.5$ & & & $285 \pm 11$ & $285 \pm 11$ & $241 \pm 34$ & \\
\hline 6583.39 & $23.6 \pm 2.0$ & $23.6 \pm 2.0$ & & & $268 \pm 11$ & $268 \pm 11$ & $214 \pm 31$ & \\
\hline 6716.42 & & & & & $9.5 \pm 2.5$ & $9.5 \pm 2.5$ & & \\
\hline 6730.72 & & & & & $11.4 \pm 2.7$ & $11.4 \pm 2.7$ & & \\
\hline 7135.8 & & & & & $17.3 \pm 4.0$ & $17.3 \pm 4.0$ & & \\
\hline 9069 & & & & & $17.5 \pm 3.8$ & $17.5 \pm 3.8$ & & \\
\hline$E(B-V)$ & $0.29 \pm 0.02$ & & & & $0.15 \pm 0.03$ & & & \\
\hline Wavelength & $\mathrm{PN} 25$ & O300 & $\mathrm{B} 600$ & U900 & PN6 & O300 & B 600 & U900 \\
\hline $3727^{\mathrm{a}}$ & \pm 65 & & & \pm 65 & $21.3 \pm 15$ & & & $21.3 \pm 15.2$ \\
\hline 3868.76 & $152 \pm 38$ & & & $152 \pm 38$ & $127 \pm 31$ & & $127 \pm 29$ & $127 \pm 31$ \\
\hline 4101.737 & $38 \pm 19$ & & & $38 \pm 19$ & $16.2 \pm 9.2$ & & & $16.2 \pm 9.2$ \\
\hline 4340.468 & \pm 48 & $180 \pm 44$ & & \pm 48 & $47 \pm 15$ & & \pm 22 & $47 \pm 15$ \\
\hline 4363.21 & \pm 48 & $84 \pm 36$ & & & $23 \pm 11$ & & \pm 22 & $23 \pm 11$ \\
\hline 4685.682 & \pm 38 & \pm 73 & & \pm 38 & $32 \pm 12$ & \pm 36 & $34 \pm 15$ & $32 \pm 12$ \\
\hline 4861.332 & $100 \pm 43$ & $100 \pm 43$ & \pm 118 & $100 \pm 27$ & $100 \pm 20$ & $100 \pm 20$ & $100 \pm 17$ & \\
\hline 4958.92 & $595 \pm 49$ & $595 \pm 49$ & $1115 \pm 547$ & & $598 \pm 23$ & $598 \pm 23$ & $438 \pm 50$ & \\
\hline 5006.85 & $1600 \pm 66$ & $1600 \pm 66$ & $3338 \pm 1626$ & & $1731 \pm 30$ & $1731 \pm 30$ & $1314 \pm 133$ & \\
\hline 5875.666 & $35.9 \pm 4.1$ & $35.9 \pm 4.1$ & \pm 102 & & $29.6 \pm 6.5$ & \pm 26 & $29.6 \pm 6.5$ & \\
\hline 6300.32 & \pm 26 & \pm 26 & & & \pm 12 & \pm 12 & & \\
\hline 6312.06 & \pm 26 & \pm 26 & & & \pm 12 & \pm 12 & & \\
\hline 6363.81 & \pm 26 & \pm 26 & & & \pm 12 & \pm 12 & & \\
\hline 6548.06 & \pm 57 & \pm 57 & \pm 93 & & \pm 15 & \pm 15 & & \\
\hline 6562.817 & $285 \pm 32$ & $285 \pm 32$ & $470 \pm 236$ & & $285 \pm 10$ & $285 \pm 10$ & & \\
\hline 6583.39 & $159 \pm 28$ & $159 \pm 28$ & $473 \pm 238$ & & $42.0 \pm 7.3$ & $42.0 \pm 7.3$ & & \\
\hline 7135.8 & \pm 46 & \pm 46 & & & \pm 12 & \pm 12 & & \\
\hline 9069 & $21.8 \pm 5.9$ & $21.8 \pm 5.9$ & & & & & & \\
\hline$E(B-V)$ & $0.31 \pm 0.10$ & & & & $0.17 \pm 0.03$ & & & \\
\hline
\end{tabular}

$\mathrm{H} \alpha / \mathrm{H} \beta$ ratio in the two cases when it was available, but used the reddening calculated from the $\mathrm{H} \gamma / \mathrm{H} \beta$ ratio otherwise. In all cases, we assumed intrinsic ratios of $I(\mathrm{H} \alpha) / I(\mathrm{H} \beta)=2.85$ and $I(\mathrm{H} \gamma) / I(\mathrm{H} \beta)=0.47$, which are appropriate for an electron temperature of $10^{4} \mathrm{~K}$ and an electron density of $10^{4} \mathrm{~cm}^{-3}$ (Osterbrock 1989). The reddening uncertainties reflect the $1 \sigma$ uncertainties in the $\mathrm{H} \alpha$ or $\mathrm{H} \gamma$ line intensities.
Note that the line intensities for PN408 in M 31 are not corrected for reddening. For this faint object, we did not detect $\mathrm{H} \gamma$, and $\mathrm{H} \alpha$ fell outside our spectral window.

Since our reddenings are based upon different line intensity ratios for different objects, we consider them in greater detail before proceeding. All of our $\mathrm{H} \alpha$ based reddenings in Tables 6 through 9 are positive. 
Table 9. Line intensities for PNe in the bulge of M 31

\begin{tabular}{|c|c|c|c|c|c|c|c|}
\hline Wavelength & PN172 & PN31 & PN80 & PN30 & PN29 & PN28 & PN23 \\
\hline 3727 & \pm 29 & \pm 30 & $41.3 \pm 8.7$ & $109 \pm 19$ & \pm 29 & \pm 43 & \pm 25 \\
\hline 3868.76 & $138 \pm 16$ & $181 \pm 13$ & $90.9 \pm 8.6$ & $108 \pm 10$ & $104 \pm 15$ & $95 \pm 16$ & $137 \pm 21$ \\
\hline & $56 \pm 10$ & $59 \pm 11$ & $38.7 \pm 8.4$ & $44 \pm 10$ & $42 \pm 10$ & $41 \pm 11$ & $52 \pm 15$ \\
\hline 4101.737 & $27.5 \pm 7.0$ & $18.0 \pm 4.7$ & $32 \pm 10$ & $29.1 \pm 5.6$ & $25.9 \pm 6.4$ & $21.8 \pm 9.0$ & $25 \pm 12$ \\
\hline & $47.0 \pm 5.4$ & $47.0 \pm 4.5$ & $47.0 \pm 5.9$ & $47.0 \pm 6.3$ & $47.0 \pm 8.6$ & $47.0 \pm 7.9$ & $47.0 \pm 9.9$ \\
\hline 4363.21 & $5.8 \pm 4.7$ & $.2 \pm 3.6$ & \pm 10 & \pm 10 & \pm 14 & \pm 8 & \pm 19 \\
\hline & \pm 6 & \pm 6 & \pm 8 & $24.1 \pm 4.0$ & $21.6 \pm 5.0$ & \pm 10 & \pm 14 \\
\hline & $100.0 \pm 5.0$ & $100.0 \pm 4.4$ & $100.0 \pm 5.0$ & $100.0 \pm 4.9$ & $100.0 \pm 6.9$ & $100.0 \pm 7.8$ & $100.0 \pm 8.5$ \\
\hline & $613 \pm 18$ & $737 \pm 21$ & $605 \pm 20$ & $645 \pm 21$ & $691 \pm 30$ & $685 \pm 32$ & $459 \pm 26$ \\
\hline 5006.85 & $319 \pm 49$ & $2253 \pm 59$ & $1869 \pm 60$ & $2063 \pm 63$ & $2219 \pm 91$ & $2151 \pm 96$ & $1347 \pm 75$ \\
\hline 5875.666 & $15.6 \pm 2.3$ & & & & & & \\
\hline$E(B-V)$ & $0.41 \pm 0.30$ & $0.40 \pm 0.25$ & $-0.16 \pm 0.33$ & $0.16 \pm 0.35$ & $-0.03 \pm 0.48$ & $0.15 \pm 0.44$ & $0.74 \pm 0.56$ \\
\hline Wavelength & PN12 & PN10 & PN1 & $\mathrm{PN} 3$ & PN38 & PN36 & PN53 \\
\hline 3727 & \pm 86 & \pm 25 & \pm 40 & \pm 11 & $18.9 \pm 7.0$ & \pm 31 & $22.6 \pm 6.2$ \\
\hline & $176 \pm 30$ & $70 \pm 12$ & $158 \pm 18$ & $64.6 \pm 6.9$ & $57.4 \pm 8.1$ & $130 \pm 15$ & $84.0 \pm 7.4$ \\
\hline 396 & $55 \pm 16$ & $33 \pm 11$ & $63 \pm 12$ & $22.5 \pm 5.6$ & $23.9 \pm 6.8$ & $103 \pm 15$ & $37.7 \pm 6.6$ \\
\hline 410 & $26 \pm 11$ & \pm 19 & $31.0 \pm 8.6$ & \pm 13 & $34.0 \pm 6.8$ & $29.4 \pm 8.8$ & $15.2 \pm 2.9$ \\
\hline & $47.0 \pm 9.9$ & $47 \pm 12$ & $47.0 \pm 8.7$ & $47.0 \pm 6.7$ & $47.0 \pm 5.9$ & $47.0 \pm 5.4$ & $47.0 \pm 4.5$ \\
\hline & $.7 \pm 8.0$ & \pm 18 & \pm 12 & \pm 12 & \pm 9 & $20.7 \pm 4.5$ & $11.8 \pm 3.4$ \\
\hline & $.2 \pm 7.7$ & \pm 15 & \pm 15 & \pm 11 & \pm 8 & \pm 7 & \pm 5 \\
\hline & $100.0 \pm 8.6$ & $100 \pm 11$ & $100 \pm 10$ & $100.0 \pm 9.1$ & $100.0 \pm 6.1$ & $100.0 \pm 5.0$ & $100.0 \pm 4.1$ \\
\hline & $586 \pm 38$ & $6 \pm 29$ & $593=$ & $672 \pm 37$ & $408 \pm 16$ & $382 \pm 13$ & $674 \pm 18$ \\
\hline & 173 & $83 \pm 81$ & $1825 \pm 109$ & $3 \pm 115$ & $1256 \pm 44$ & $1136 \pm 35$ & $2062 \pm 52$ \\
\hline & $.6 \pm 3.6$ & $25.3 \pm 7.1$ & & $59.3 \pm 11.0$ & & $8.8 \pm 1.6$ & $38.2 \pm 5.3$ \\
\hline$E(B-V)$ & $0.88 \pm 0.56$ & $0.18 \pm 0.68$ & $0.49 \pm 0.49$ & $-0.55 \pm 0.38$ & $-0.09 \pm 0.33$ & $0.78 \pm 0.30$ & $0.31 \pm 0.25$ \\
\hline Wavelength & PN52 & PN42 & PN45 & $\mathrm{PN} 43$ & PN48 & PN95 & PN47 \\
\hline 3727 & \pm 63 & \pm 19 & $26.1 \pm 7.7$ & \pm 14 & \pm 10 & $19.7 \pm 6.1$ & $19.5 \pm 7.5$ \\
\hline & $63 \pm 12$ & $122.2 \pm 8.0$ & $120.5 \pm$ & $56.7 \pm 6.9$ & $125.9 \pm 5.6$ & \pm 3.4 & $9 \pm 5.0$ \\
\hline & $2 \pm 10$ & $.9 \pm 8.0$ & & & $7 \pm 5.8$ & \pm 4.8 & $4 \pm 6.6$ \\
\hline & & \pm 4.1 & & & \pm 2.9 & $23.4 \pm$ & $21.6 \pm 4.5$ \\
\hline & $47.0 \pm 9.9$ & $0 \pm 4.3$ & $47.0 \pm 3.6$ & $47.0 \pm 6.5$ & \pm \pm 3.3 & $47.0 \pm 3.4$ & $47.0 \pm 4.4$ \\
\hline & \pm 13 & $25.9 \pm 3.7$ & $27.2 \pm 3.2$ & \pm 11 & $11.7 \pm 2.6$ & \pm 5 & $8.1 \pm 3.2$ \\
\hline & $2.2 \pm 6.7$ & \pm 6 & \pm 4 & \pm 7 & $4.0 \pm 1.9$ & \pm 3 & \pm 4 \\
\hline & $100.0 \pm 8.2$ & $100.0 \pm 4.7$ & $100.0 \pm 3.4$ & $100.0 \pm 4.9$ & \pm 3.3 & $.0 \pm 2.4$ & $0 \pm 3.6$ \\
\hline & $464 \pm 27$ & \pm 1 & & & \pm 14 & \pm 5.8 & $4 \pm 10$ \\
\hline 50 & $1364 \pm 75$ & $1 \pm 54$ & $1381 \pm 27$ & \pm 52 & $0 \pm 43$ & $998 \pm 15$ & $1452 \pm 28$ \\
\hline & \pm 6 & $12.0 \pm 1.8$ & $15.9 \pm 1.6$ & $34.1 \pm 6.7$ & $36.1 \pm 3.7$ & $18.6 \pm 1.5$ & $20.9 \pm 2.4$ \\
\hline$E(B-V)$ & $0.63 \pm 0.56$ & $0.30 \pm 0.24$ & $0.44 \pm 0.20$ & $-0.72 \pm 0.36$ & $-0.25 \pm 0.19$ & $0.11 \pm 0.19$ & $0.09 \pm 0.25$ \\
\hline Wavelength & PN408 & PN93 & PN92 & PN97 & PN91 & PN387 & PN380 \\
\hline 3727 & & $39.1 \pm 8$ & & \pm 17 & \pm 30 & $126 \pm 17$ & \pm 26 \\
\hline & & \pm 6 & & & $8 \pm 9.9$ & & $108 \pm 10$ \\
\hline 396 & & $27.3 \pm 5.4$ & $44.8 \pm 8.5$ & 4.3 & $29.5 \pm 8.1$ & $43.9 \pm$ & $51.8 \pm 9.6$ \\
\hline & & $21.9 \pm 4.8$ & & $21.7 \pm 4.5$ & \pm 14 & $30.3 \pm 6.2$ & $28.8 \pm 6.8$ \\
\hline 4340.468 & & $47.0 \pm 8.7$ & $47.0 \pm 5.0$ & $51.6 \pm 4.4$ & $47.0 \pm 6.0$ & $47.0 \pm 3.5$ & $50.4 \pm 5.7$ \\
\hline 4363.2 & & \pm 16 & $18.4 \pm 4.1$ & $9.3 \pm 3.4$ & $18.9 \pm 4.8$ & $12.3 \pm 2.7$ & $30.5 \pm 5.1$ \\
\hline & & $15.0 \pm 3$ & \pm 6 & \pm 4 & \pm 8 & \pm 2.7 & \pm 5 \\
\hline & $100 \pm 29$ & $100.0 \pm 5.4$ & & $100.0 \pm 3.2$ & $100.0 \pm 5.3$ & $100.0 \pm 4.0$ & $100.0 \pm 4.6$ \\
\hline & $491 \pm 85$ & $673 \pm 24$ & & $565 \pm 13$ & $482 \pm 17$ & $525 \pm 15$ & $614 \pm 22$ \\
\hline 5006.85 & $1431 \pm 242$ & $2070 \pm 72$ & $1703 \pm 42$ & $1744 \pm 35$ & $1437 \pm 44$ & $1591 \pm 36$ & $1851 \pm 48$ \\
\hline 5875.666 & & $30.0 \pm 6.0$ & $17.2 \pm 1.8$ & $22.7 \pm 2.4$ & & $16.1 \pm 1.3$ & $18.3 \pm 1.5$ \\
\hline 6548.06 & & & & $12.0 \pm 2.1$ & & & $4.4 \pm 1.8$ \\
\hline 6562.817 & & & & $285.0 \pm 6.4$ & & & $285.0 \pm 7.3$ \\
\hline 6583.39 & & & & $35.8 \pm 2.5$ & & & $16.9 \pm 2.0$ \\
\hline$E(B-V)$ & & $-0.24 \pm 0.49$ & $0.51 \pm 0.28$ & $0.02 \pm 0.02$ & $0.26 \pm 0.34$ & $0.39 \pm 0.20$ & $0.20 \pm 0.02$ \\
\hline
\end{tabular}


The overwhelming majority of our $\mathrm{H} \gamma$-based reddenings in Table 9 are also either positive or consistent with no reddening, but our $1 \sigma \mathrm{H} \gamma$ line intensity uncertainties do allow negative reddenings in four cases (PN3, PN43, PN48, and $\mathrm{PN53}$ ). We considered not using $\mathrm{H} \gamma$ to determine the reddening, but rejected this option for four reasons. First, for the four planetary nebulae in M 32 for which we measured an $\mathrm{H} \gamma$ intensity from the $\mathrm{B} 600$ spectrum, the reddeningcorrected $\mathrm{H} \gamma$ intensity has the expected value of approximately $47 \%$ that of $\mathrm{H} \beta$ after correcting for reddening using the $\mathrm{O} 300 \mathrm{H} \alpha$ intensity. In these four cases, then, $\mathrm{H} \alpha$ and $\mathrm{H} \gamma$ would yield similar reddenings. Second, our ultimate aim is to calculate electron temperatures and oxygen abundances from these line intensities. If we measured the intensity of $[\mathrm{O}$ III $] \lambda 4363$ relative to $\mathrm{H} \gamma$ and $[\mathrm{O}$ III $] \lambda \lambda 4959$, 5007 relative to $\mathrm{H} \beta$, and assumed $I(\mathrm{H} \gamma) / I(\mathrm{H} \beta)=0.47$, we would obtain final intensities for the [O III] lines that would be statistically indistinguishable from those obtained by correcting for reddening using the $\mathrm{H} \gamma$ intensity. Applying a negative reddening correction does affect the oxygen abundance we derive by reducing the $[\mathrm{O}$ II $] \lambda 3727$ intensity, but this effect has less impact on the oxygen abundance than the uncertainty in the electron temperature since there is so little oxygen in the form of $\mathrm{O}^{+}$. Third, forcing $I(\mathrm{H} \gamma) / I(\mathrm{H} \beta)=0.47$ via a reddening correction, even if negative, accounts for any errors in the sensitivity calibration that might otherwise systematically affect the [O III] lines and the subsequent oxygen abundances. Fourth, on average, our $\mathrm{H} \alpha$ - and $\mathrm{H} \gamma$-based reddenings agree. The mean $\mathrm{H} \alpha$-based reddening for all objects (both M 31 and M 32) is $E(B-V)=0.18 \pm 0.04 \mathrm{mag}$, while the mean $\mathrm{H} \gamma$-based reddening for all of the planetary nebulae in the bulge of M 31 is $E(B-V)=0.18 \pm 0.08 \mathrm{mag}$, if negative reddening values are included, or $E(B-V)=$ $0.25 \pm 0.06 \mathrm{mag}$, if negative reddening values are set to zero (the uncertainties are the standard errors in the means). Thus, the reddenings computed from $\mathrm{H} \alpha$ and $\mathrm{H} \gamma$ are similar. For comparison, the foreground reddening to M 31 is $E(B-V)=0.093 \pm 0.009 \mathrm{mag}$ (mean of McClure \& Racine 1969; van den Bergh 1969; and Burstein \& Heiles 1984). It is not surprising that the mean reddening for the planetary nebulae is $0.10 \mathrm{mag}$ greater than the foreground value, for planetary nebulae suffer additional reddening due to internal dust and dust within M 31 and M 32 . Consequently, we have chosen to correct for "reddening" even when $E(B-V)$ is negative.

\section{Oxygen abundances}

Tables 4 and 5 present the electron temperatures and the oxygen abundances for the planetary nebulae in M 32 and in the bulge of $\mathrm{M} 31$, respectively. We only observed two ionization stages of oxygen, $\mathrm{O}^{+}$and $\mathrm{O}^{++}$. We accounted for unseen stages in our oxygen abundance calculations using the ionization correction factors (ICF) computed according to the prescription of Kingsburgh \& Barlow
(1994), which employs the line intensities of He II $\lambda 4686$ and He I $\lambda 5876$ to correct for unseen ionization stages of oxygen. Further details may be found in Stasińska et al. (1998). Tables 4 and 5 present two oxygen abundance calculations. The abundances in Col. 3 are simply the sum of the $\mathrm{O}^{+}$and $\mathrm{O}^{++}$ionic abundances. The abundances in Col. 4 are those from Col. 3 corrected for the ICF. The ICF is normally small because He II $\lambda 4686$ is weak. The oxygen abundances in Col. 4 will be adopted in future work.

In calculating the oxygen abundances, we assumed an electron density of $4000 \mathrm{~cm}^{-3}$ in all cases. With electron densities of $1 \mathrm{~cm}^{-3}$ and $210^{4} \mathrm{~cm}^{-3}$, the oxygen abundance changes by a maximum of $-0.02 \mathrm{dex}$ and $+0.07 \mathrm{dex}$, respectively, for the planetary nebulae in M 31, and by a maximum of -0.03 dex and +0.11 dex, respectively, for the planetary nebulae in M 32.

In instances where only upper limits to intensities were available, we adopted the following approach. When we had upper limits for the intensities of the helium lines these limits were used to calculate the ICF. If we did not observe He I $\lambda 5876$ (because it was outside our spectral window), we made no correction for unseen stages of oxygen regardless of the intensity of He II $\lambda 4686$. (Only in two cases, PN29 and PN30 in M 31, did we detect He II $\lambda 4686$ when He I $\lambda 5876$ was outside our spectral window.) When we only had an upper limit to [O III $\lambda 4363$, we used this to derive an upper limit to the electron temperature, and this temperature limit was then used to derive a lower limit to the oxygen abundance. In these instances, we did not compute an error for either the electron temperature or the oxygen abundance, and have indicated the results listed in Tables 4 and 5 as limits. When we had an upper limit for [O II $] \lambda 3727$, we adopted this limiting intensity for the line. In this case, the $\mathrm{O}^{+}$ionic abundance is over-estimated, but its contribution to the total oxygen abundance was normally small.

Our uncertainties for the electron temperatures and oxygen abundances reflect the uncertainties in the [O III] line intensities alone. As noted earlier, reddening introduces a further uncertainty through its effect upon [O II] $\lambda 3727$, but this has less influence upon the oxygen abundance than the uncertainty in the electron temperature. The electron temperature uncertainty that we quote is simply the temperature range permitted by the $(1 \sigma)$ limiting values of the [O III $]$ line intensities. Similarly, our oxygen abundance uncertainties are derived from the abundances calculated using the extreme values of the electron temperature.

\section{Discussion}

In Tables 4 and 5, we derive oxygen abundances for approximately half of the planetary nebulae we observed. For the rest, we derive lower limits. Many of the oxygen abundance limits, however, are very useful. Six of the fourteen 
temperature limits in Table 5 are below $10^{4} \mathrm{~K}$, and one is even below $9000 \mathrm{~K}$. If we separate the planetary nebulae in Table 5 on the basis of whether they have temperatures or temperature limits, the mean oxygen abundances of the two sets differ at the $92 \%$ confidence level, with the set of objects with temperature limits having a higher mean oxygen abundance by at least 0.11 dex. Table 5 shows clearly that we are able to measure oxygen abundances up to approximately the solar value $(12+\log (\mathrm{O} / \mathrm{H})=8.93 \mathrm{dex}$; Anders \& Grevesse 1989). Since the Loral3 CCD has only modest sensitivity at [O III $] \lambda 4363$, the quantum efficiency being about $22 \%$, these results are by no means the limit of what is possible with $4 \mathrm{~m}$-class telescopes.

In several companion papers, we shall exploit the spectroscopic observations of planetary nebulae in M 32 and in the bulge of M 31 in several ways. First, we intend to study the evolution of the planetary nebulae in these galaxies relative to those in the Milky Way and the Magellanic Clouds. We also intend to investigate the chemical evolution of M 32 and the bulges of M 31 and the Milky Way individually as well as the chemical evolution of DHGs as a class.

Acknowledgements. We would like to thank George Jacoby for making several very helpful comments concerning a preliminary version of this paper, and for making further helpful suggestions as referee. MGR would like to thank the Natural Sciences and Engineering Research Council of Canada and Marshall McCall for their financial support while this research was being done. MGR also thanks the Physics and Astronomy Department at York University for its hospitality and computing facilities while this work was being finished. MLM thanks the Natural Sciences and Engineering Research Council of Canada for its continuing support.

\section{References}

Aller L.H., 1987, Physics of Thermal Gaseous Nebulae. Dordrecht: D. Reidel Publishing Co

Anders E., Grevesse N., 1989, Geochim. Cosmochim. Acta 53, 197

Anderson E., 1987, Reduction of Long-Slit Spectroscopic Data Using IRAF, IRAF User Handbook, Vol. 2B. Tucson: National Optical Astronomy Observatory

Bender R., Burstein D., Faber S.M., 1992, ApJ 399, 462

Bender R., Burstein D., Faber S.M., 1993, ApJ 411, 153

Brocato E., Matteucci F., Mazzitelli I., Tornambè A., 1990, ApJ 349, 458

Burstein D., Heiles C., 1984, ApJS 54, 33

Ciardullo R., Jacoby G.H., Ford H.C., Neill J.D., 1989, ApJ 339,53

Ford H.C., Jenner D.C., 1975, ApJ 202, 365

Ford H.C., Jacoby G. H., Jenner D.C., 1978, ApJ 223, 94

Forestini M., Charbonnel C., 1997, A\&AS 123, 241

Henry R.B.C., 1989, MNRAS 241, 453

Iben I. Jr., Renzini A., 1983, ARA\&A 21, 271

Kingsburgh R.L., Barlow M.J., 1994, MNRAS 271, 257

Kormendy J., 1985, ApJ 295, 73

Le Fèvre O., Crampton D., Felenbok P., Monnet G., 1994, A\&A 282, 325

Massey P., Valdes F., Barnes J., 1992, A User's Guide to Reducing Spectra with IRAF, IRAF User Guide, Vol. 2B. Tucson: National Optical Astronomy Observatory

McCall M.L., 1984, MNRAS 208, 253

McCall M.L., Rybski P.M., Shields G.A., 1985, ApJS 57, 1

McClure R.D., Racine R., 1969, AJ 74, 1000

Osterbrock D.E., 1989, Astrophysics of Gaseous Nebulae and Active Galactic Nuclei. Mill Valley: University Science Books

Perinotto M., 1991, ApJS 76, 687

Richer M.G., 1993, ApJ 415, 240

Schild R.E., 1977, AJ 82, 337

Stasińska G., Richer M.G., McCall M.L., 1998, A\&A 366, 667

van den Bergh S., 1969, ApJS 19, 145

Wheeler J.C., Sneden C., Truran J.W.Jr., 1989, ARA\&A 27, 279 


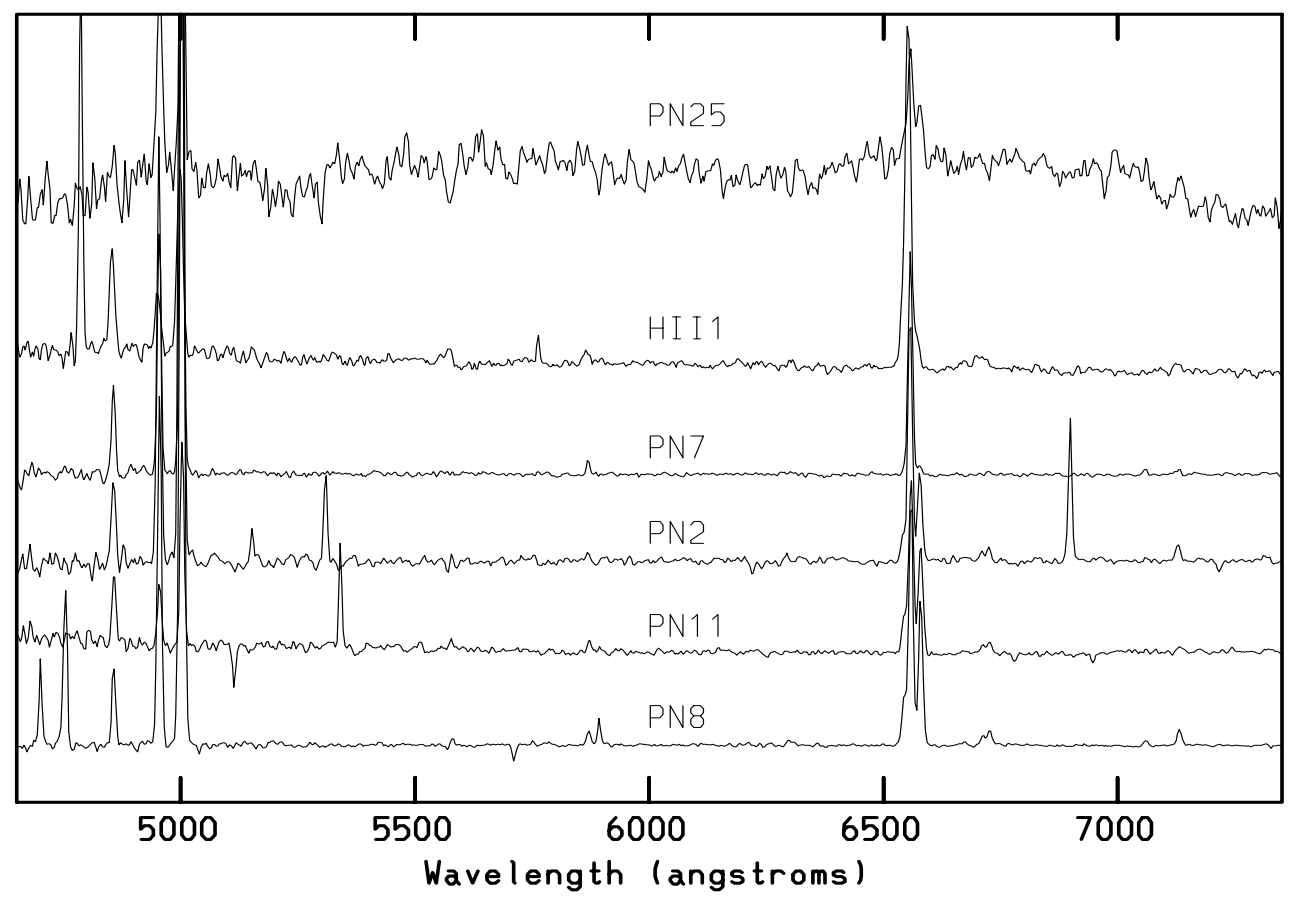

Fig. 1. The O300 spectra for PN8, PN11, PN2, PN7, Hil 1, and PN25 in the M 32 field. The spectra are displayed such that $\mathrm{H} \beta$ spans the entire free intensity scale. Consequently, lines stronger than $\mathrm{H} \beta$ overlap in adjacent spectra. We show only the spectral range blueward of $7350 \AA$. In all of the spectra we present, cosmic rays were not removed unless they interfered with measuring line intensities, so many obviously remain. PN25 is very close to M 32's nucleus, so the sky subtraction is poorer for this object. H II 1 is an H II region in the background disk of M 31 (Ford \& Jenner 1975)

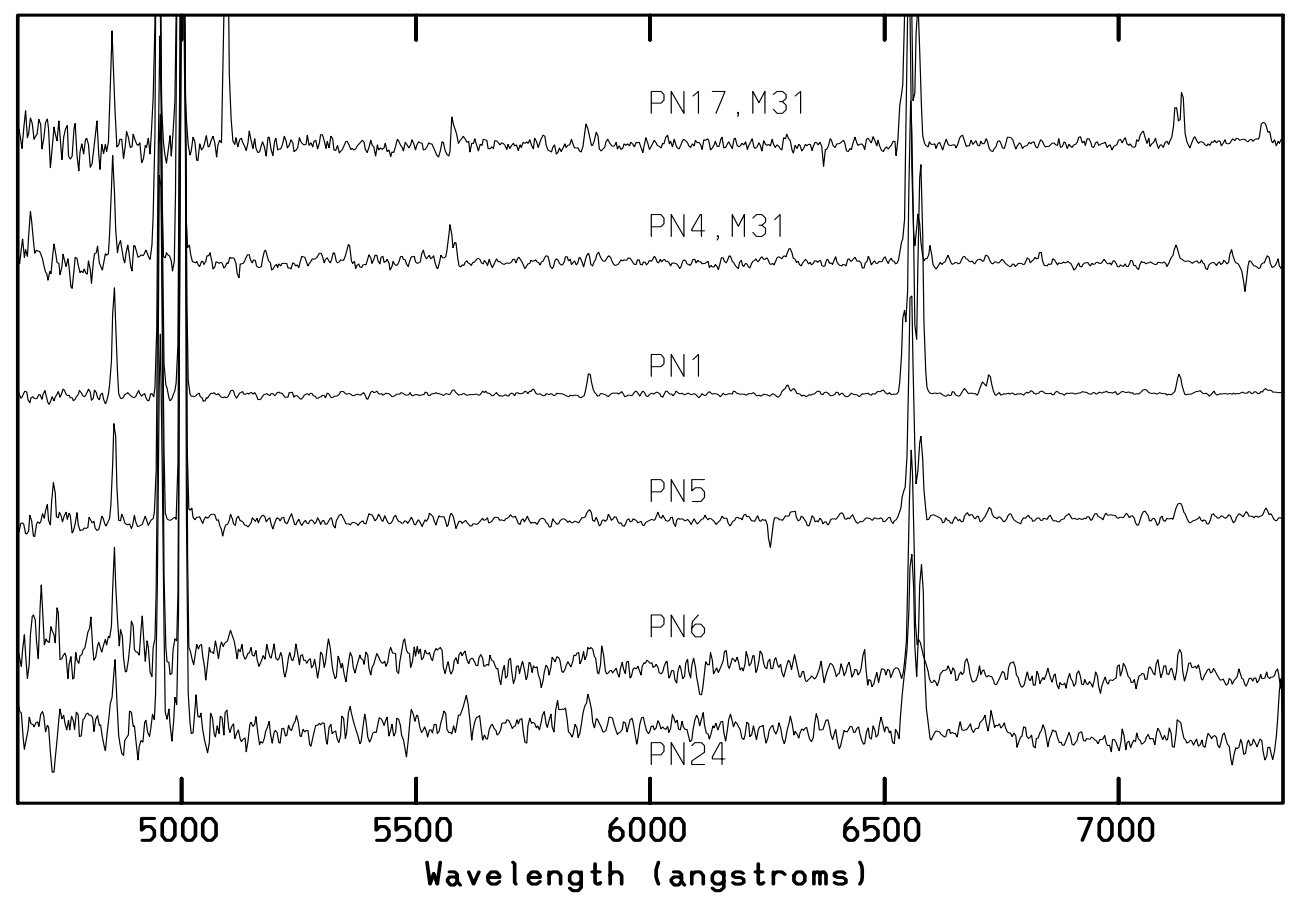

Fig. 2. The O300 spectra for PN24, PN6, PN5, PN1, PN4, and PN17 in the M 32 field. The format is identical to Fig. 1. Like PN25, PN24 is also very close to M 32's nucleus and suffers from somewhat poorer background subtraction. Note that PN4 and PN17 are background planetary nebulae in the disk of M 31 (Ford \& Jenner 1975) 


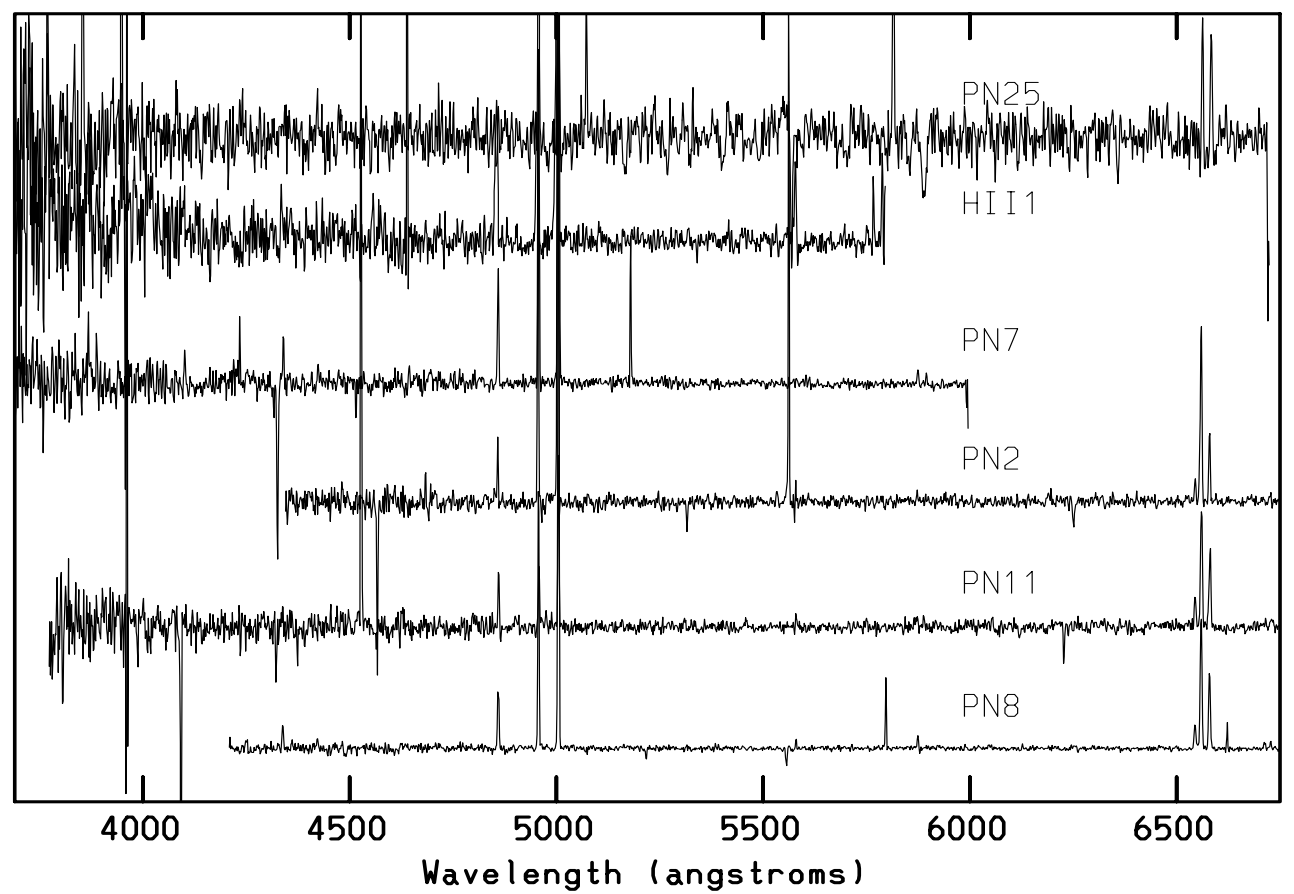

Fig. 3. The B600 spectra for PN8, PN11, PN2, PN7, H II 1, and PN25 in the M 32 field. For PN8, PN11, and PN25, the scaling is such that $\mathrm{H} \alpha$, not $\mathrm{H} \beta$, defines the free intensity range. The full useful wavelength range of the spectra, $3690 \AA$ to $6750 \AA$, is shown. See Fig. 1 for comments on individual objects

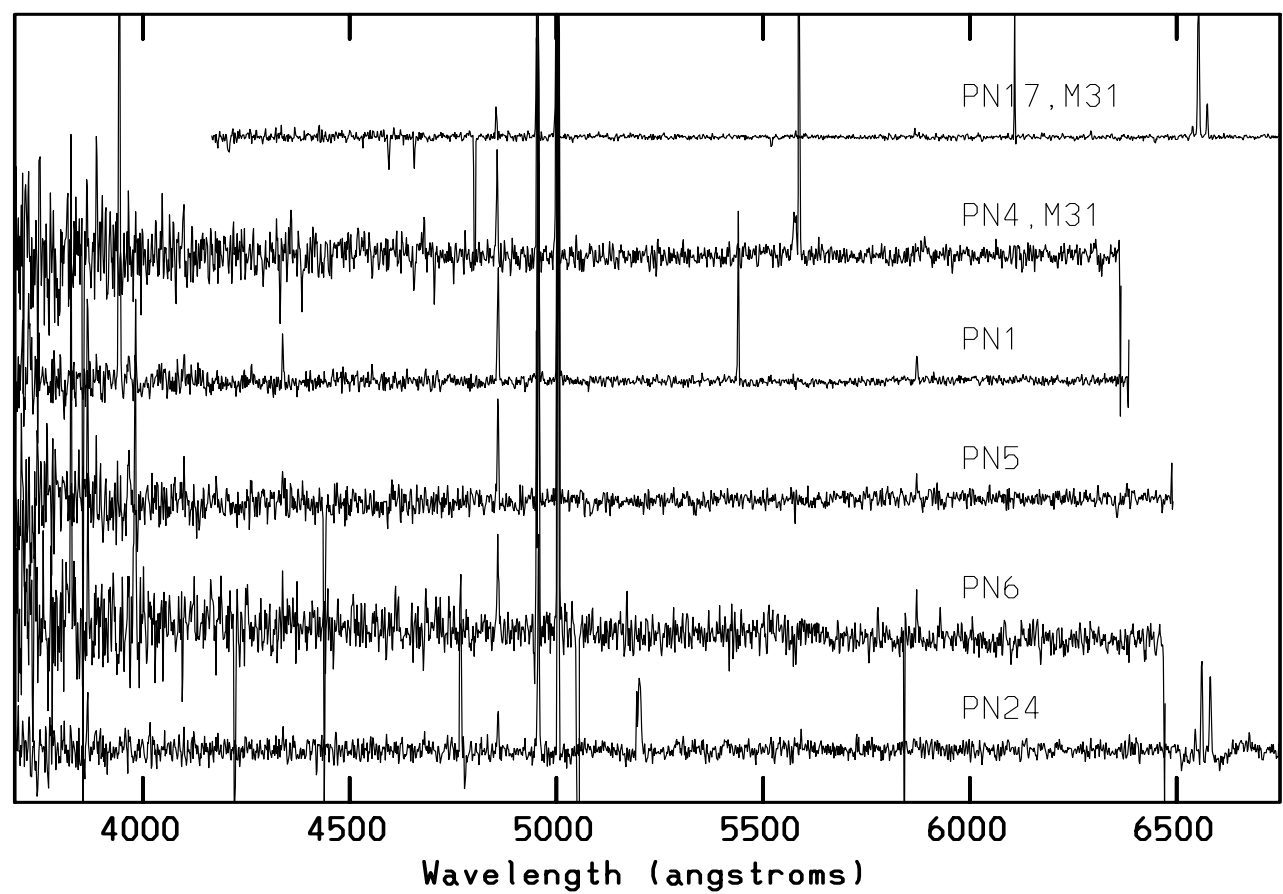

Fig. 4. The B600 spectra for PN24, PN6, PN5, PN1, PN4, and PN17 in the M 32 field. For PN24 and PN17 (M 31), H $\alpha$, and not $\mathrm{H} \beta$, defines the free intensity range. The full useful wavelength range of the spectra, $3690 \AA$ to $6750 \AA$, is shown. See Fig. 2 for comments on individual objects 


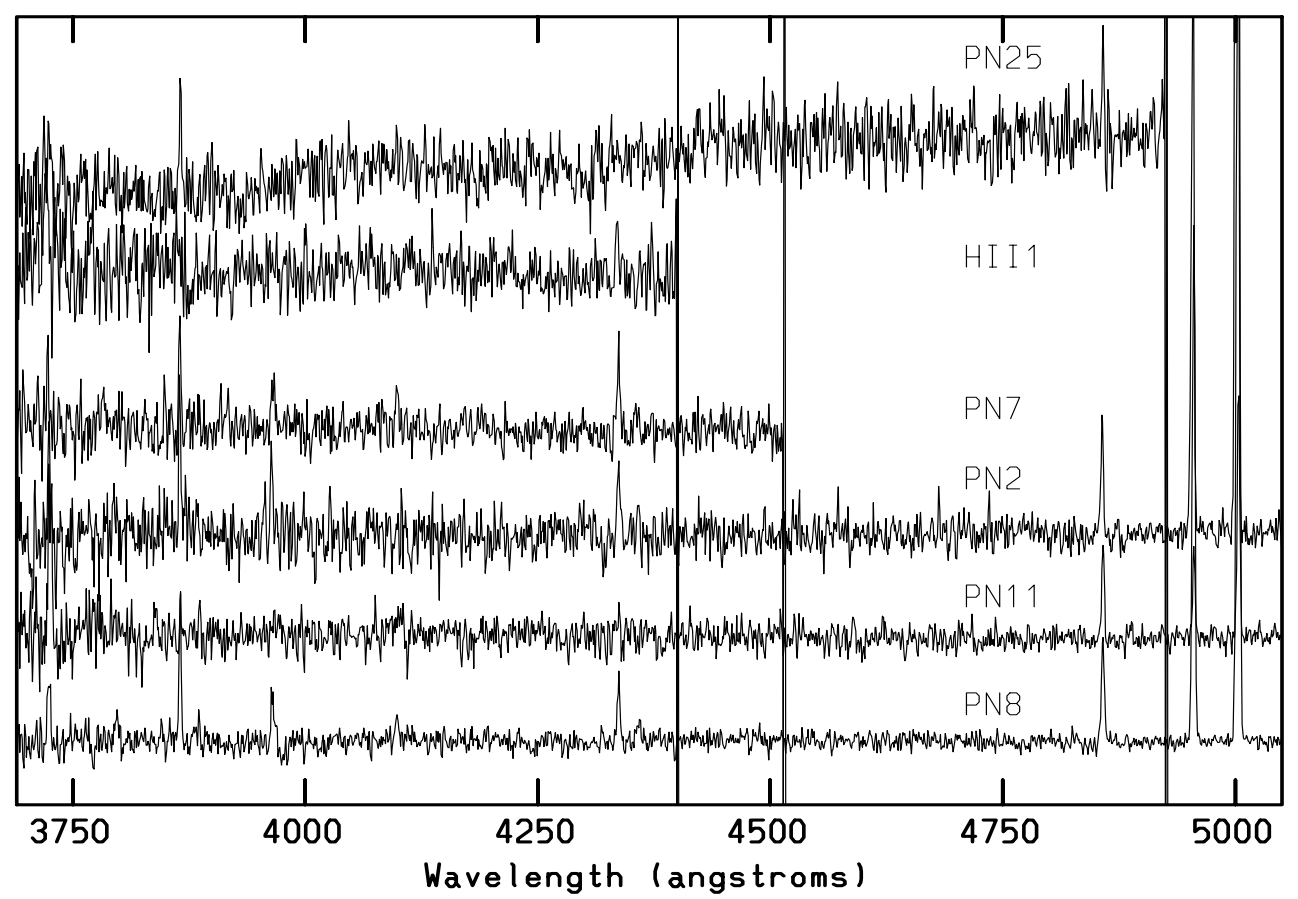

Fig. 5. The U900 spectra for PN8, PN11, PN2, PN7, Hir 1, and PN25 in the M 32 field. Only for PN11 and PN25 does H $\beta$ define the full intensity scale. For the other objects, the full intensity scale is defined by $\mathrm{H} \gamma$. The full useful wavelength range, $3690 \AA$ to $5050 \AA$, is displayed. See Fig. 1 for comments on individual objects

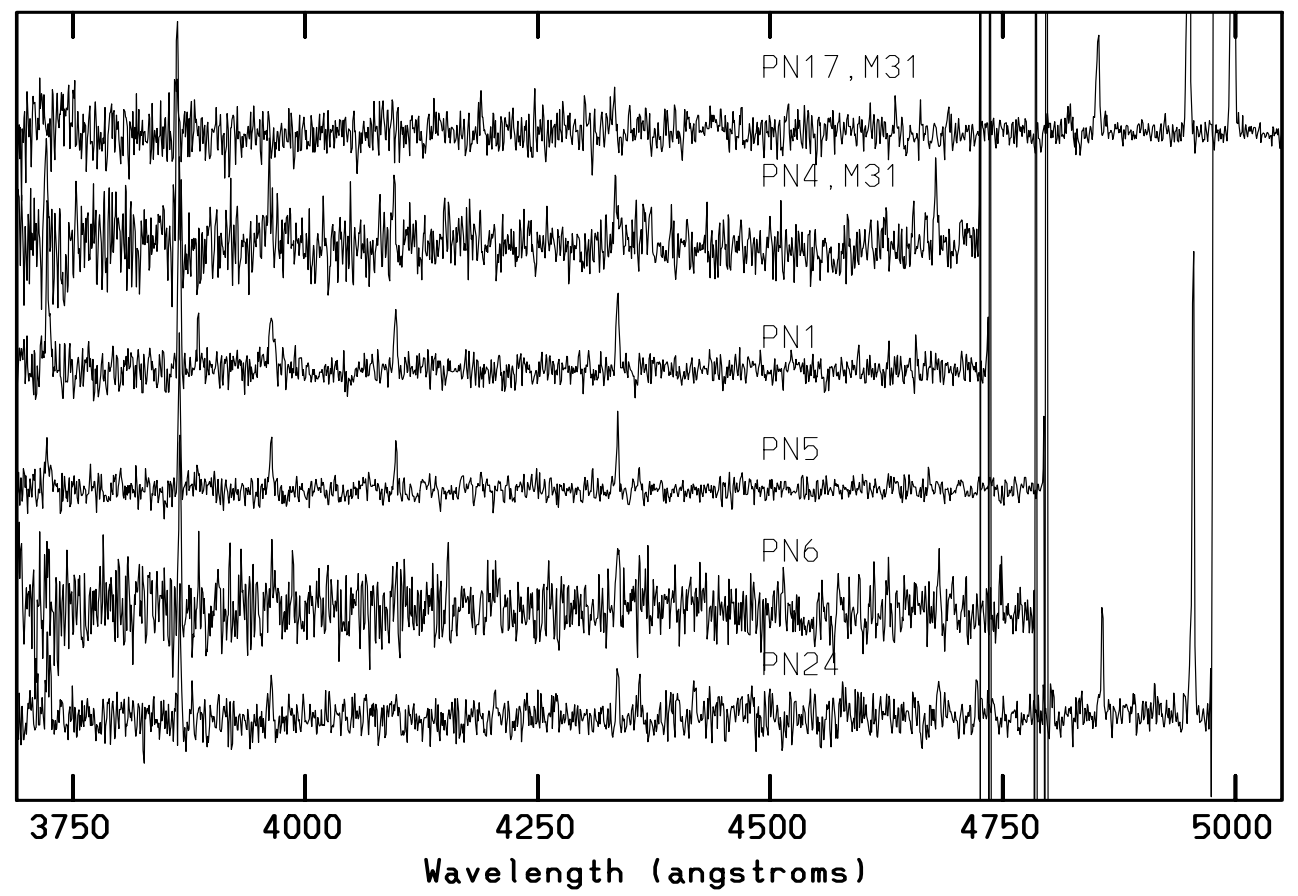

Fig. 6. The U900 spectra for PN24, PN6, PN5, PN1, PN4, and PN17 in the M 32 field. $\mathrm{H} \beta$ defines the full intensity scale for PN24 and PN17, but $\mathrm{H} \gamma$ does so for the other objects. The full useful wavelength range, $3690 \AA$ to $5050 \AA$, is displayed. See Fig. 2 for comments on individual objects 


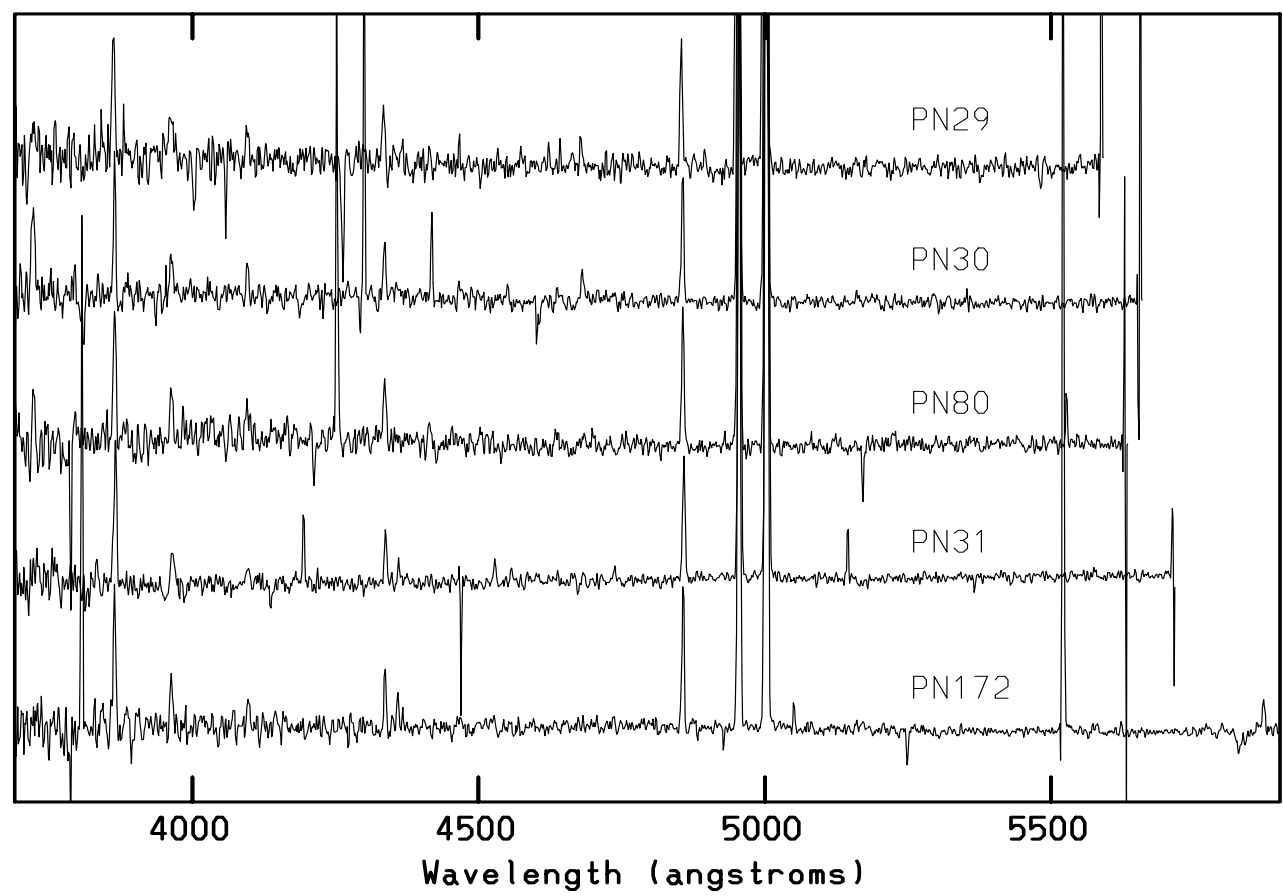

Fig. 7. The B600 spectra for PN172, PN31, PN80, PN30, and PN29 in the M 31 bulge field. The intensity scaling is set so that $\mathrm{H} \beta$ occupies the full free intensity scale in all cases, and the entire useful wavelength range is shown

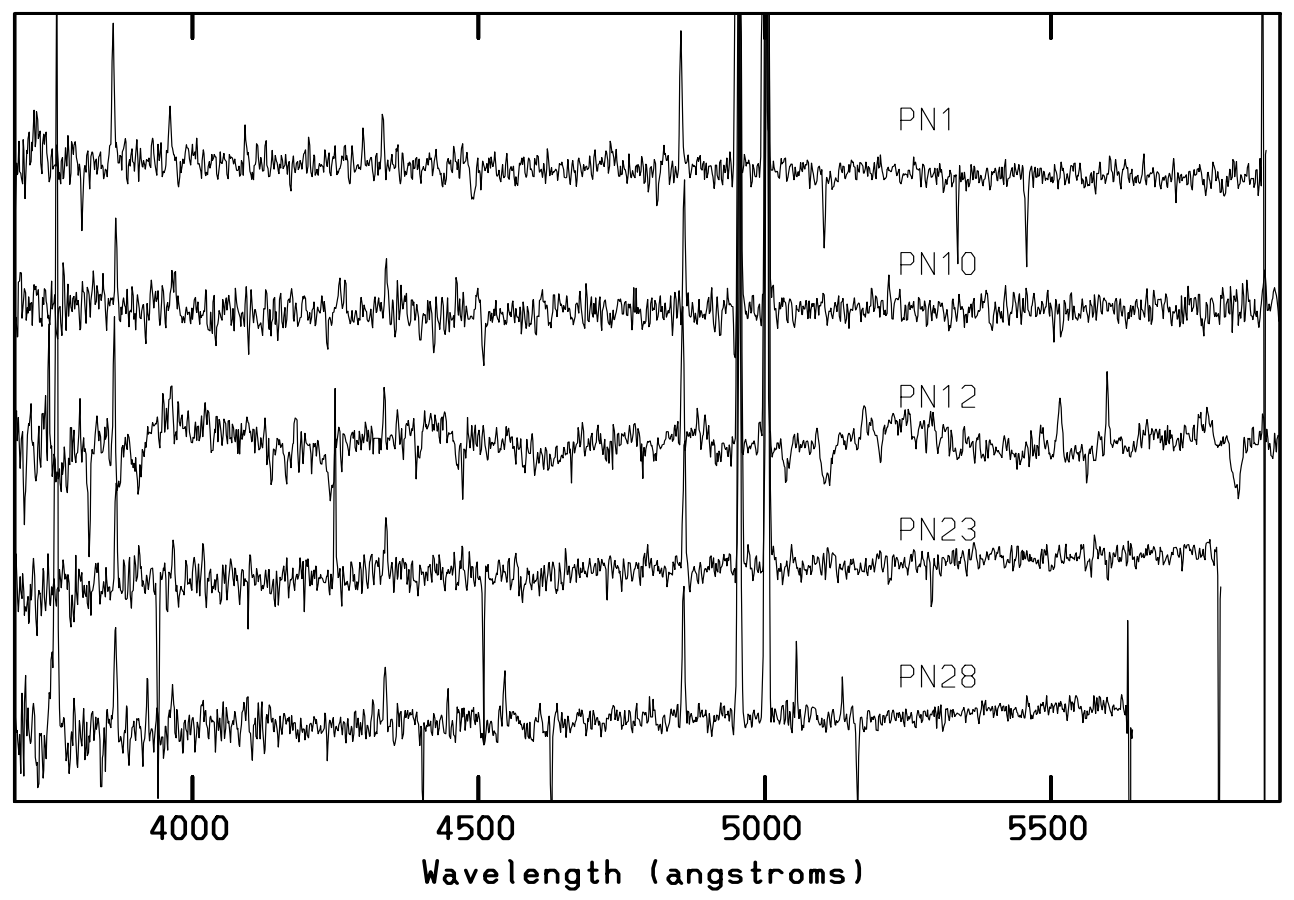

Fig. 8. The B600 spectra for PN28, PN23, PN12, PN10, and PN1 in the M 31 bulge field. The intensity and wavelength scales are as in Fig. 7. Note that the background subtraction is poorer for PN12 than is normally the case 


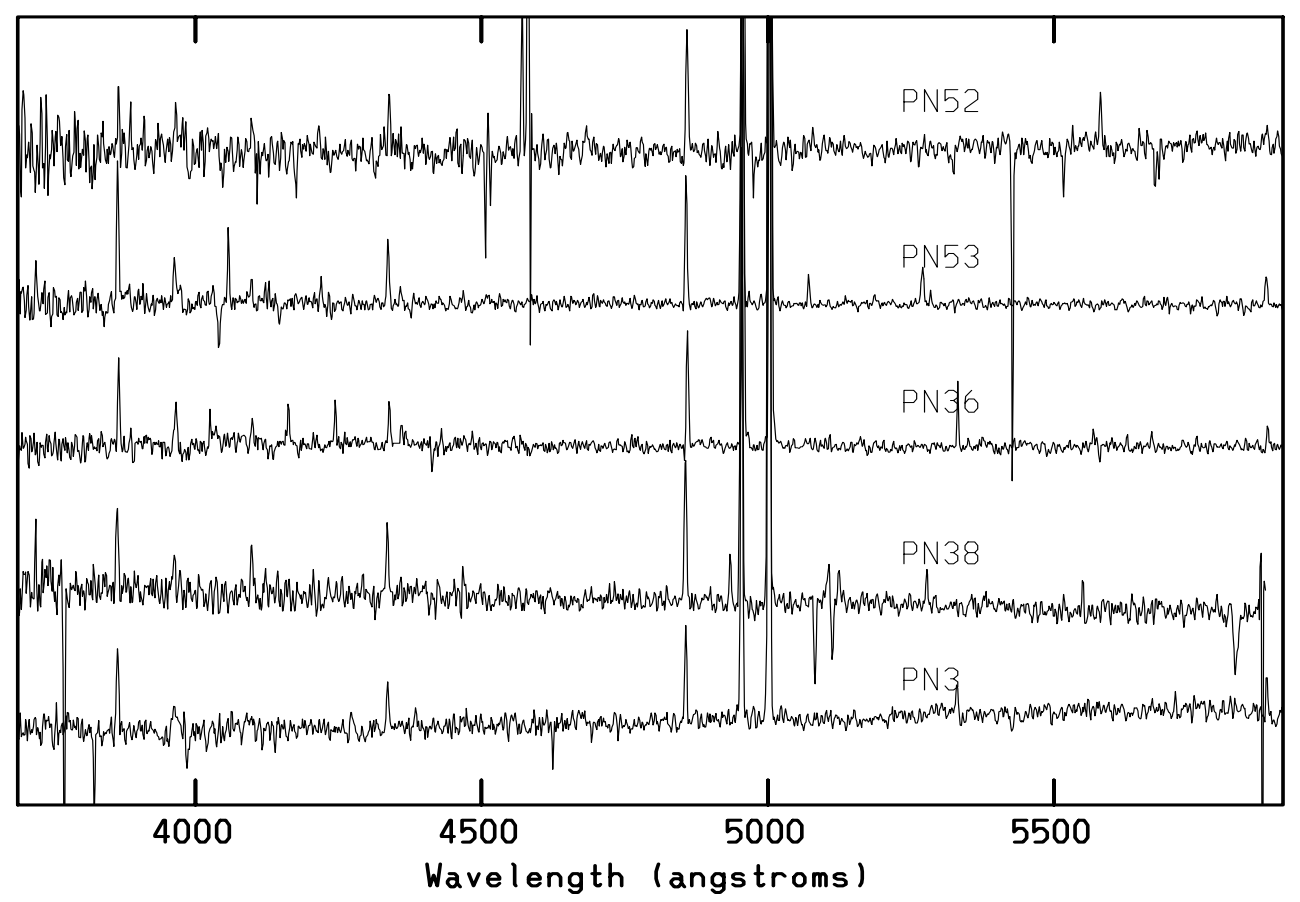

Fig. 9. The B600 spectra for PN3, PN38, PN36, PN53, and PN52 in the M 31 bulge field. The intensity and wavelength scales are as in Fig. 7

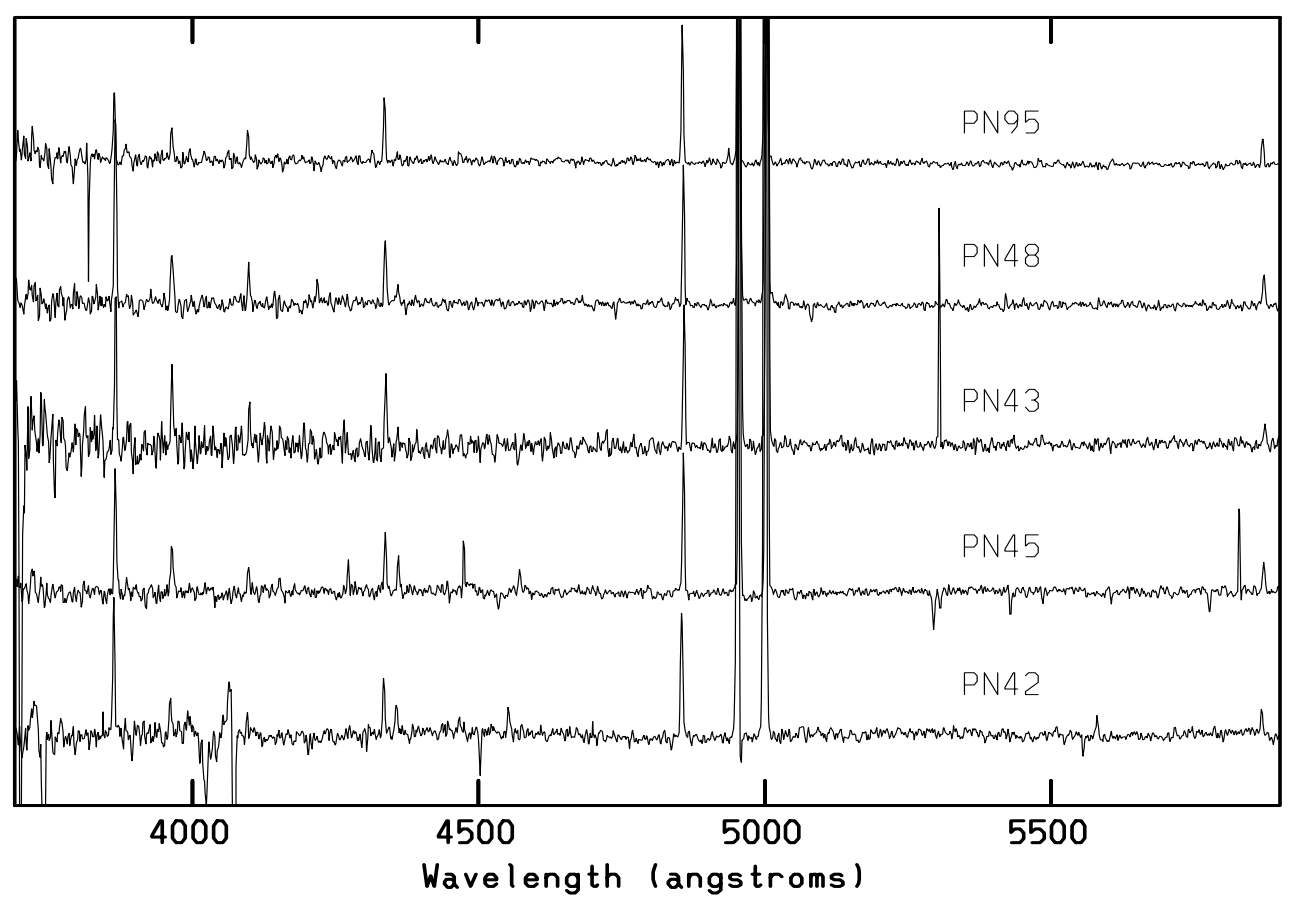

Fig. 10. The B600 spectra for PN42, PN45, PN43, PN48, and PN95 in the M 31 bulge field. The intensity and wavelength scales are as in Fig. 7 


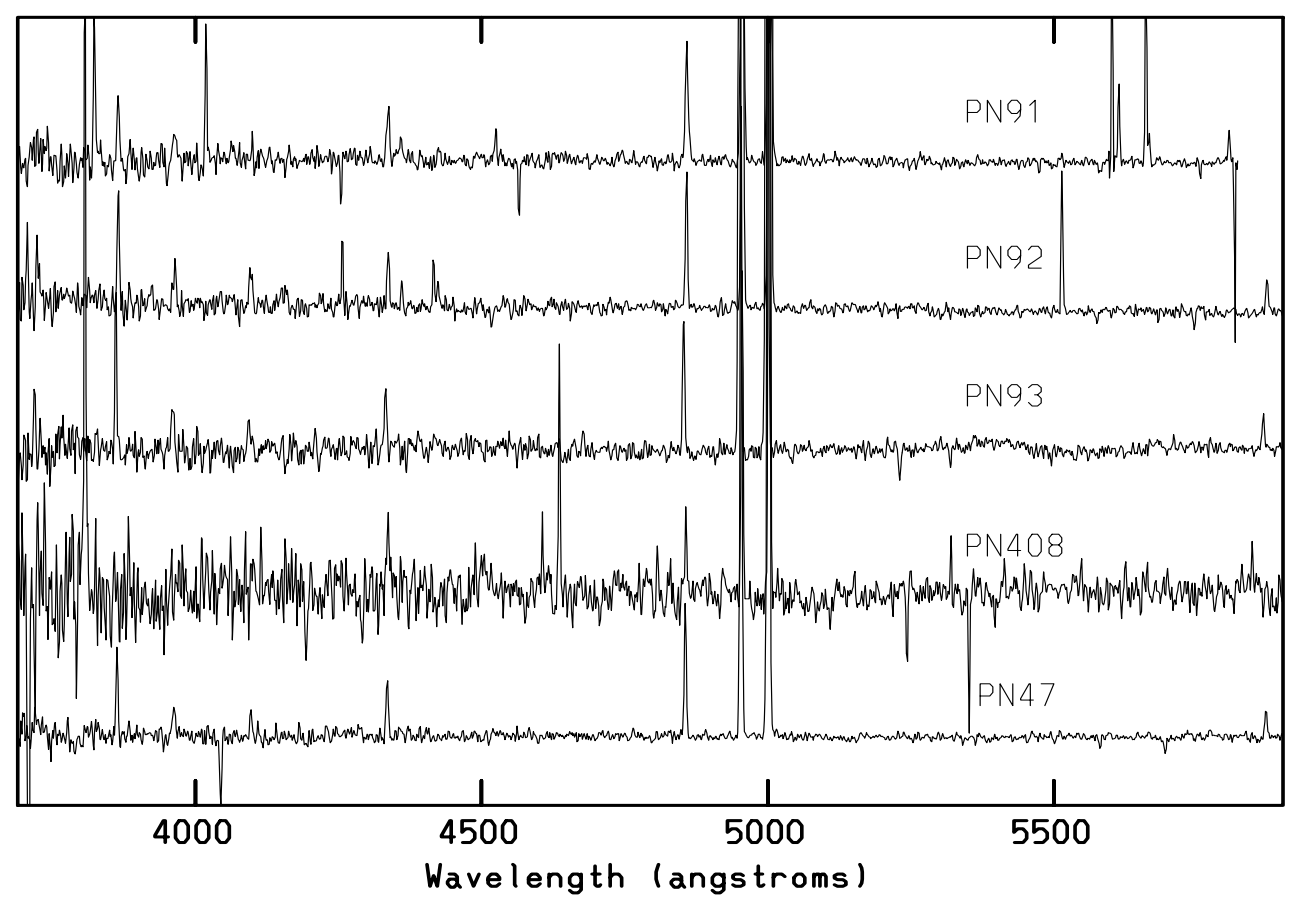

Fig. 11. The B600 spectra for PN47, PN408, PN93, PN92, and PN91 in the M 31 bulge field. The intensity and wavelength scales are as in Fig. 7. Note that the signal-to-noise is poor for the very faint object PN408

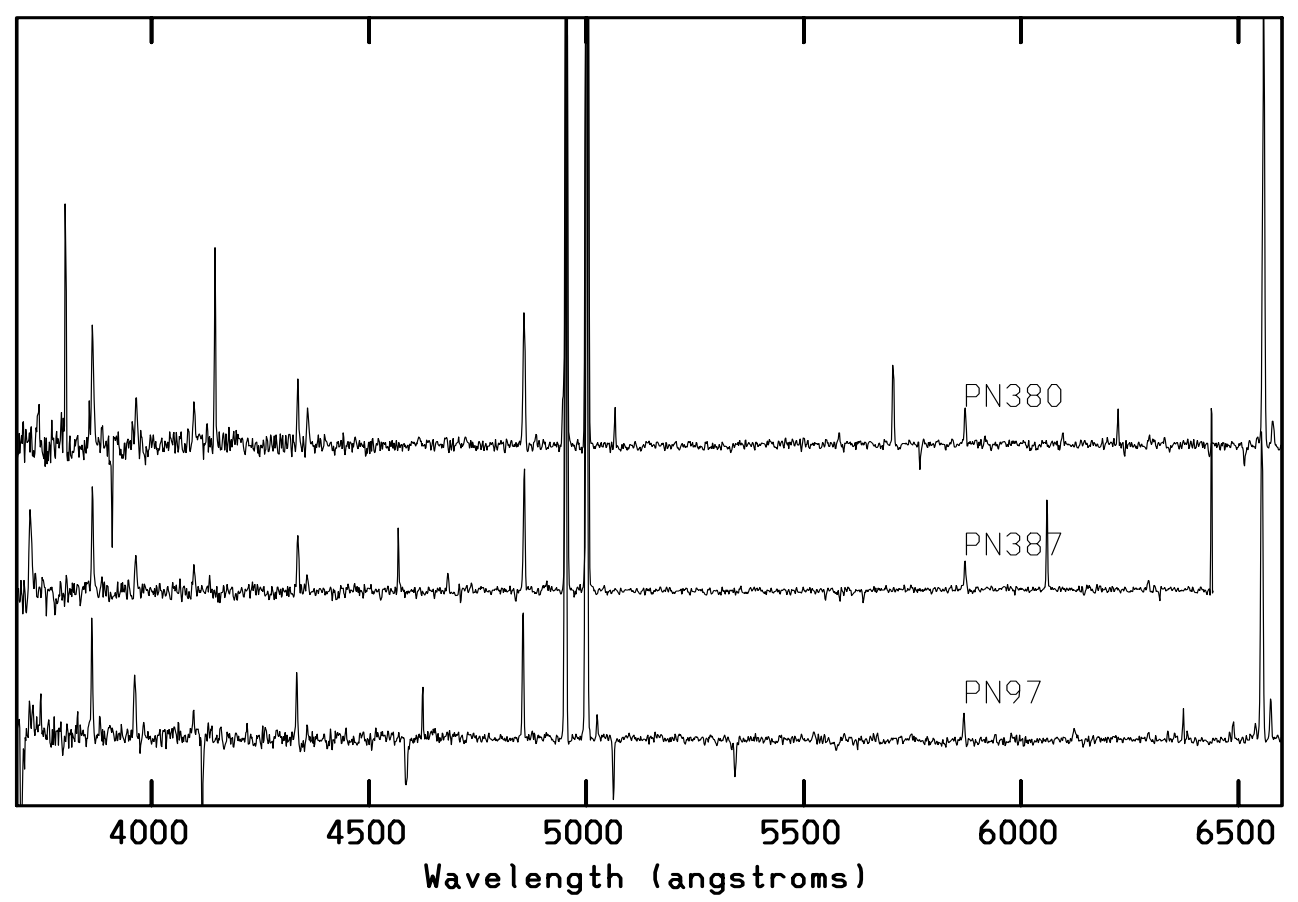

Fig. 12. The B600 spectra for PN97, PN387, and PN380 in the M 31 bulge field. The intensity scaling is set so that H $\beta$ occupies the full free intensity scale in all cases, and the entire useful wavelength range is shown. For PN97 and PN380, though, the wavelength range extends to $6600 \AA$ 